\title{
Research on the Construction of a Petrophysical Model of a Heterogeneous Reservoir in the Hydrate Test Area in the Shenhu Area of the South China Sea (SCS)
}

\author{
Xing Lei, ${ }^{1,2}$ Liu Xueqin $\mathbb{D}^{\text {, }},{ }^{3,4,5}$ Liu Huaishan, ${ }^{1,2}$ Qin Zhiliang, ${ }^{3,4,5}$ and Ma Benjun ${ }^{3,4,5}$ \\ ${ }^{1}$ Key Lab of Submarine Geosciences and Prospecting Techniques, MOE, Ocean University of China, \\ Qingdao Shandong 266100, China \\ ${ }^{2}$ Laboratory of Marine Mineral Resource, Pilot National Laboratory for Marine Science and Technology, \\ Qingdao Shandong 266071, China \\ ${ }^{3}$ Acoustic Science and Technology Laboratory, Harbin Engineering University, Harbin 150001, China \\ ${ }^{4}$ Key Laboratory of Marine Information Acquisition and Security (Harbin Engineering University), Ministry of Industry and \\ Information Technology, Harbin 150001, China \\ ${ }^{5}$ College of Underwater Acoustic Engineering, Harbin Engineering University, Harbin 150001, China
}

Correspondence should be addressed to Liu Xueqin; liuxq@hrbeu.edu.cn

Received 21 February 2021; Revised 11 May 2021; Accepted 30 September 2021; Published 31 October 2021

Academic Editor: Wei Zhang

Copyright (c) 2021 Xing Lei et al. This is an open access article distributed under the Creative Commons Attribution License, which permits unrestricted use, distribution, and reproduction in any medium, provided the original work is properly cited.

\begin{abstract}
The occurrence characteristics of hydrates in the Shenhu area reflect a typical inhomogeneity in terms of spatial distribution. It is difficult to accurately describe the petrophysical properties of a reservoir using a petrophysical model considering a single cementation factor parameter. According to the analysis of a mathematical model and the estimation results of $V_{\mathrm{p}}$ and $V_{\mathrm{s}}$, the unique structure of foraminiferal sediment particles provides opportunities for forming a diversified hydrate occurrence in the foraminiferal area. In areas where hydrates are thin and interbedded, hydrate reservoirs are generally three-phase media, with obvious thermoelastic properties. Therefore, the parameters of the three characteristic models of the pore-filling model, particle cementation model, and thermodynamic elastic model are all included in the correction model. The weights of the influence factors are then changed to realize an accurate description of the petrophysical characteristics of the correction model in different drilling areas and at different formation depths, reducing the limitations of using a single petrophysical model to describe the petrophysical characteristics of heterogeneous regions under the influence of multiple factors.
\end{abstract}

\section{Introduction}

A petrophysical model is an equivalent model that describes the microscopic characteristics of rock. It links the microscopic characteristics and macroscopic elastic properties of the rock pore structure $[1,2]$. Therefore, constructing a reasonable and accurate petrophysical model is the key to analyzing the reservoir structure and evaluating the total energy amount through seismic methods [3]. The physical properties of rock mainly depend on three aspects: the rock composition, the rock microstructure, and the thermodynamic environment in which it is located $[3,4]$. The currently applicable rock physics models are mainly divided into three types: (1) equivalent volume-average models, such as the Voigt-Reuss-Hill (VRH) model and Hashin-Shtrikman (HS) model. This type of model assumes an average internal stress in the rock and is suitable for static isotropy; it can be used to constrain upper and lower limits of the model physical property parameters to improve the inversion accuracy, but it is difficult to apply to the inversion of physical property parameters in complex reservoirs $[5,6] ;$ (2) equivalent models based on self-adaptation, differentiation, and scattering theory, such as the Kuster-Toksoz (KT) model and Differential Equivalent Medium (DEM) model. This type of model is mainly used to study the internal pore structure of the rock and the influence of pore filling on the rock 
properties. However, there are many model parameters and the calculation process is complicated; (3) equivalent model of saturated rock, such as the Gassmann equation, Gassmann anisotropy form based on the Gassmann equation, and $\mathrm{Xu}-$ White (XW) model suitable for argillaceous sandstone. The Gassmann equation assumes more ideal conditions and is currently widely used in the estimation of porosity and saturation of hydrate reservoirs, but this model does not consider the influence of pore shape [8-10]. Moreover, Simplified Three-Phase Equation (STPE) model and effective medium theory (EMT) model also belong to the category of equivalent model of saturated rock. The STPE is a simple model, and the EMT is based on the first criterion of physics. The simple model simplifies the factors that affect hydrate saturation and lacks a rigorous theoretical basis. Although the EMT is rigorous in theory, it involves many parameters and the value is difficult to determine, thus adding new uncertain factors $[11,12]$. In addition, a thermoelastic model is suitable for simulating the existence of a high proportion of free gas. The thermoelastic model evolves from the saturated rock model, taking into account the elastic petrophysical characteristics of the thermoelastic medium. However, the scope of application of this model is relatively narrow; it is only applicable to formations with large heat flows. As the structural geology and lithology change, most formations show heterogeneous characteristics. It is difficult to choose only one of the petrophysical models to describe the microscopic petrophysical characteristics of an entire region $[13,14]$.

Natural gas hydrate is an ice-like crystalline substance formed by natural gas and water under high-pressure and low-temperature conditions. According to the influencing factors of the petrophysical model, the construction of the petrophysical model of a gas hydrate reservoir needs to clarify its characteristic microparameters: (1) composition of the rock [15]. The composition of the rock matrix is complex and changeable. Determining the content of each component is an important condition for the accurate construction of a rock model. Researchers often use borehole data as constraints to ensure estimation accuracy; (2) internal structure of the rock [16]. The internal structure of the rock is mainly related to the occurrence state of the hydrate and the form of the cementation; different occurrence states and cementation forms of hydrate reservoirs correspond to different petrophysical properties; how to determine the cementation mode in a complex reservoir environment is the key and difficult point of model construction. Therefore, it is difficult to accurately determine the appropriate rock skeleton before the model is constructed. In addition, hydrate reservoirs are different from other oil and gas reservoirs. Due to the existence of free gas, they are more sensitive to temperature and pressure conditions [17]. Therefore, the author infers that it is necessary to consider their thermodynamic properties, when studying the petrophysical properties of hydrate reservoirs.

The Shenhu area of the SCS is currently the main hotspot for research on highly saturated silty hydrate reservoirs, and the hydrate reservoirs in this area have strong heterogeneity $[4,13,16]$. According to the rock matrix type, the hydrate reservoirs in the Shenhu area can be divided into foraminifera-control-type, quartz-control-type, and clay- control-type reservoirs $[17,18]$. Different types of organic molecular structures will also lead to different physical parameters of hydrate-bearing sedimentary rocks [1]. According to the cementing method, these structures can be divided into particle-supported structures, skeletonsupported structures, and other types of structures (Figure 1). According to the occurrence mode, the organic matter can be divided into a pore-filling type and fracture type. According to the type of organic molecules forming hydrates and the source of free gas, hydrate reservoirs can be divided into structure I, structure II, and structure $\mathrm{H}$ hydrates. Therefore, in view of the heterogeneity of the hydrate reservoirs in the Shenhu area, it is important to construct a petrophysical model suitable for the study of the physical properties of the hydrate reservoirs in a study area.

In this paper, the hydrate reservoir in the trial mining area in the Shenhu area is taken as the research object, and regional geological and logging data are comprehensively analyzed. Based on effective medium theory and two-phase medium theory, a pore-filling model, particle cementation model, and thermoelastic model are used to create a petrophysical model. This kind of petrophysical model is used as a reference to describe the quantitative relationship between elastic parameters such as the $V_{\mathrm{p}}, V_{\mathrm{s}}$, and reservoir microscopic parameters such as porosity, hydrate saturation, gas saturation, and pore microstructure. Based on the $V_{\mathrm{p}}$ of borehole data, density data are used as a constraint to establish the best petrophysical model suitable for the Shenhu area of the SCS and to further determine the $V_{\mathrm{s}}$ in the study area based on the petrophysical model, providing data support for petrophysical parameter inversion of the hydrate reservoir in the Shenhu test mining area of the SCS.

\section{Geology of the Study Area}

The Shenhu area in the northern part of the SCS is located in the middle section of the northern slope of the SCS (Figure 2). It is currently a hotspot for natural gas hydrate exploration in China [19]. The hydrate layer in this area has obvious inhomogeneity and discontinuity in the vertical and lateral directions in terms of the spatial distribution of the reservoir, the occurrence state of the hydrate, and the type of reservoir sediment. The development of extensional faults, folds, and diapir structures in the study area opened up favorable pathways for the migration of natural gas from the deeper strata to the shallower strata and determined the discontinuous state of the spatial distribution of the hydrate reservoirs [20]. For example, the multiaxial discontinuity of BSR is obvious seismic profile evidence of lateral inhomogeneity distribution of hydrate reservoirs (Figure 2).

In terms of sedimentation, the Shenhu area is dominated by semideep sea sediments rich in calcareous ultramicrofossils, accompanied by terrigenous clastic materials; to ascertain the sedimentation of the natural gas hydrates in the northern slope of the SCS, the Guangzhou Marine Geological Survey has carried out three offshore drilling expeditions in the Shenhu area. The drilling results show that the saturation of hydrate is as high as $40 \%$. In 2007, physical samples 


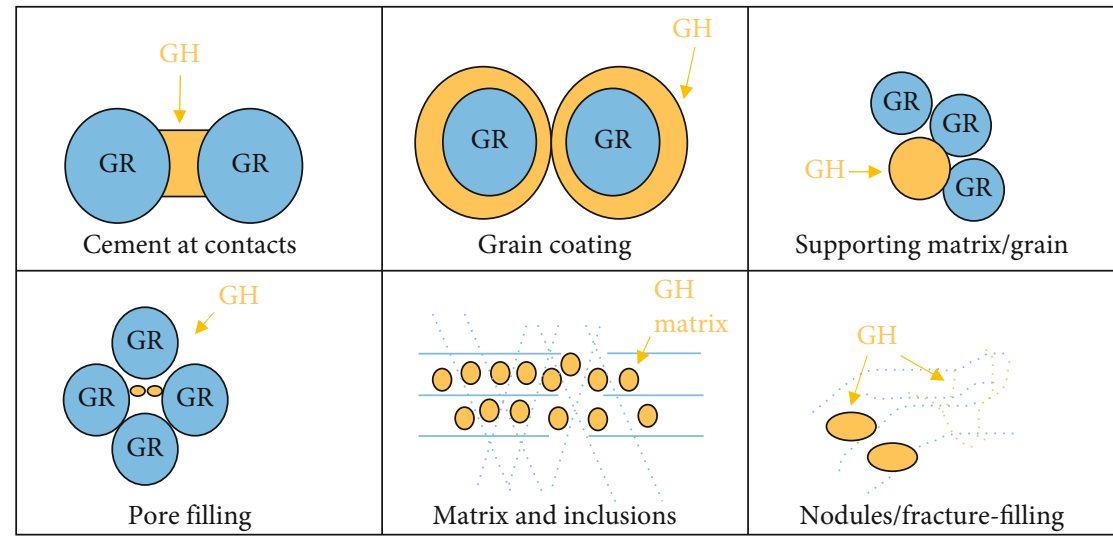

Figure 1: Occurrence status of hydrate in the Shenhu area [14].

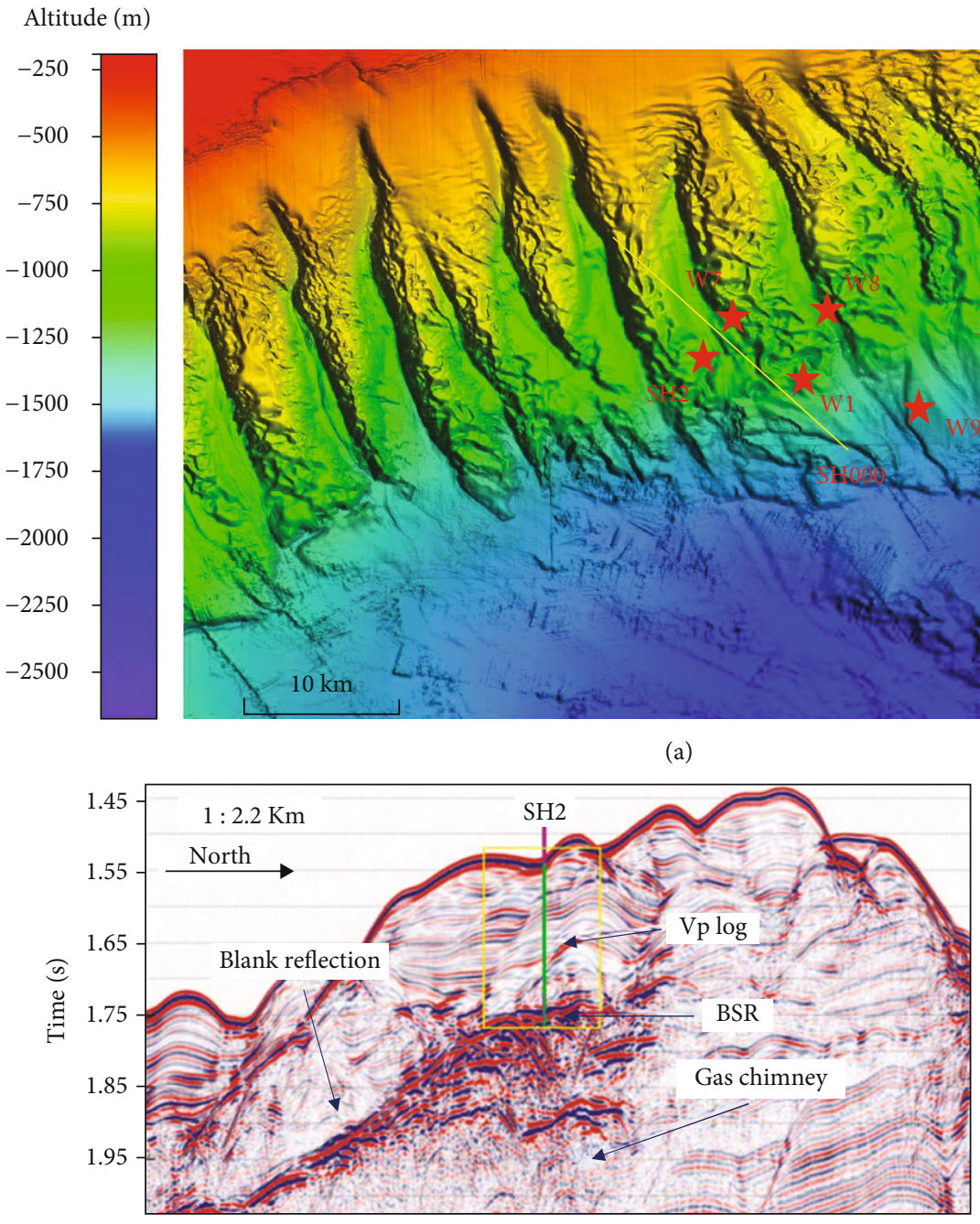

(b)

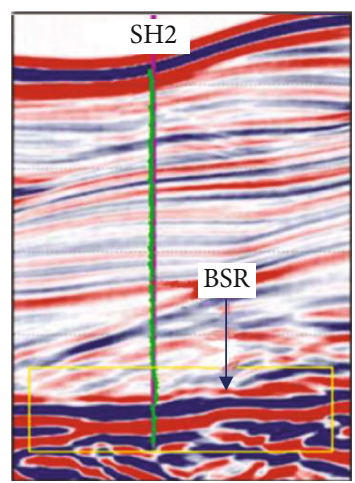

(c)

Figure 2: Study area in the Shenhu area [2]: (a) water depth and location of the study area; (b) seismic section SH000 in the work area; (c) zoom of the seismic section SH000.

of natural gas hydrate were successfully drilled and recovered at sites $\mathrm{SH} 2, \mathrm{SH} 3$, and $\mathrm{SH} 7$, which were distributed in layers in unconsolidated fine-grained clay or sandy clay in a uniformly dispersed state [21]. In the core collected at site W19 in 2015, the SCS sediments were rich in foraminifera, which not only increased the content of the coarse-grained 
matrix particles but also provided a large amount of sedimentary pore space due to its porous characteristics [22]. Yang et al. [19] divided the hydrates in Shenhu into five occurrence states, namely, thick-layered hydrate, dispersed hydrate, porphyritic hydrate, hydrate near a fault, and thin-layered hydrate (Figure 1).

In addition, the existence of free gas migration pathways such as those along diapirs and faults has led to certain differences in the heat flow values $[23,24]$. For example, the geothermal gradients estimated based on the in situtemperature measurements at sites W07, W08, and W09 $\left(102-111^{\circ} \mathrm{C} / \mathrm{km}\right)$ are higher than those at the background site W01 $\left(65^{\circ} \mathrm{C} / \mathrm{km}\right)$. So some scholars inferred that the structural characteristics of the geology in this area control the temperature conditions [25]. Therefore, there are differences in the hydrate occurrence areas of the Shenhu area in terms of the rock matrix composition, cementation method, thermoelastic characteristics, etc. To achieve a detailed quantitative description of a hydrate reservoir, a reasonable rock physics model must be constructed.

\section{Logging Data Analysis}

In this paper, well SH2, well W17, and well W19 are selected as the research objects, and the drilling positions are indicated in Figure 2. The borehole data were obtained in 2007 and 2015, the $V_{\mathrm{p}}$ data sampling interval was $0.02 \mathrm{~m}$, and the borehole depth was $200-300 \mathrm{~m}$. In addition, the seismic data considered in this work are from a seismic section closest to site $\mathrm{SH} 2$ and the gas hydrate can be indicated by BSR. The seismic data features and acquisition methods are as follows: on June 7-14, 2015, a stereoscopic detection campaign was carried out in the Shenhu area with Struggle No. 4 and the Treasure Ship (equipped with single-source, singlecable marine horizontal streamers, OBSs, and vertical cables) by the Guangzhou Marine Geological Survey. A total of 11 OBS sites and 2 vertical cable sets were installed, and 24 lines of multichannel seismic surveys were completed over a total distance of $369.7 \mathrm{~km}$. The layout of the work area is shown in Figure 2. For the horizontal streamers, a unilateral receiving observation system was adopted with a single-sided cannon and 360 pathways, and the fold number was 45 .

To ascertain the occurrence and distribution of natural gas hydrates in the continental slope area of the northern SCS, the GMGS-1 expedition led by the Guangzhou Marine Geological Survey conducted offshore drilling in Shenhu in 2007, and a total of 5 sites were sampled: sites SH1, SH2, SH3, SH5, and SH7. Physical samples of natural gas hydrate were successfully drilled at sites $\mathrm{SH} 2, \mathrm{SH} 3$, and $\mathrm{SH} 7$; these hydrates were distributed in layers in a uniformly dispersed state in unconsolidated fine-grained clay or sandy clay [21]. In 2015, a third gas hydrate drilling campaign (GMGS-3) was implemented in the sea area, and the logging data of 19 sites all indicated gas hydrate, and core was collected at wells W11, W17, W18, and W19 [19]. A thick hydrate layer with a high hydrate saturation was observed at well W19, making this layer one of the best prospects for hydrate exploitation in the SCS [26].
Figure 3 shows the five logging curves from site $\mathrm{SH} 2$. Based on the characteristics of the change in the $V_{\mathrm{p}}$ curve, the thickness of the reservoir is approximately $30 \mathrm{~m}$ (190$220 \mathrm{~m}$ ), the average velocity of the overlying hydrate layer is approximately $1900 \mathrm{~m} / \mathrm{s}$, the average velocity of the hydrate reservoir $(190-220 \mathrm{~m})$ is $2070 \mathrm{~m} / \mathrm{s}$, and the maximum velocity of the hydrate reservoir is $2200 \mathrm{~m} / \mathrm{s}$. The strata above and below the hydrate layer have a $V_{\mathrm{p}}$ of $1890 \mathrm{~m} / \mathrm{s}$, which is low and likely indicates a free gas zone. According to the structural analysis of the area, the low-speed anomaly area corresponds to the lower gas source migration pathways, and the upward movement of a large amount of fluid causes a local decrease in formation velocity. The hydrate reservoirs near the well are mostly solid, liquid, and gaseous three-phase media: the solid rock is mainly composed of clay, sand, and silt, among which the main mineral components of the sand and silt are quartz and calcite, respectively. The fluid phase is mainly composed of water and gas. The analysis of the gas composition in the sediments shows that the hydrate type in the drilling area is structure I hydrate, the main gas in the sediments is hydrocarbons, and the average content of methane is $98 \%$ [7]. The rock matrix of the reservoir is mainly fine-grained clay and silt, its cementation method is pore filling, and hydrate is used as a pore-filling medium to participate in the formation of the rock skeleton.

At well W17, the increases in the resistivity and velocity logs from 210 to $250 \mathrm{mbsf}$ indicate that gas hydrate is present within this interval (Figure 4). The resistivity log from 258 to $270 \mathrm{mbsf}$ is also elevated, but the velocity log reflects a more complex interbedded rock with alternating thin beds of higher and lower velocity. Within this section, the density-porosity log values are higher than those of the neutron-porosity log, which suggests that free gas may be present in this interval [9]. At well W17, however, structure II gas hydrate is inferred to exist above and below the I-BSR based on the presence of propane (Figure 4) in the pressure core samples collected at depths of $222 \mathrm{mbsf}, 241 \mathrm{mbsf}$, and 263 mbsf [2].

The sediments observed at W19 are mainly marine finegrained sediments, mainly clayey silt and silty sand according to logging curves of gamma ray (Figure 5) and scholars' research results [27]. The high content of calcite is mainly derived from calcareous ultrafine fossils with foraminifera as the main body. For foraminifera-controlled hydrate reservoirs, hydrates are present in thick layers. Among the sediment components of this type of reservoir, the sand content of foraminifera-controlled hydrate-bearing rock is significantly greater, up to $10.85 \%$ greater, and the main contributor of sand is foraminifera; the calcite content is $50 \%$ greater, and the clay content is significantly decreased [28]. The above research shows that due to the increase in the content of coarse components such as sand and silt, the overall grain size of this layer is coarse, and the sorting is poor. In addition, the weak sedimentary compaction and the deposition of foraminifera fossils result in the high porosity and permeability of this reservoir (Figures 6-8). The shell structure of foraminifera in this sediment not only increases the intergranular voids of the sediments but also produces intragranular pores [18]. These reservoir structures 

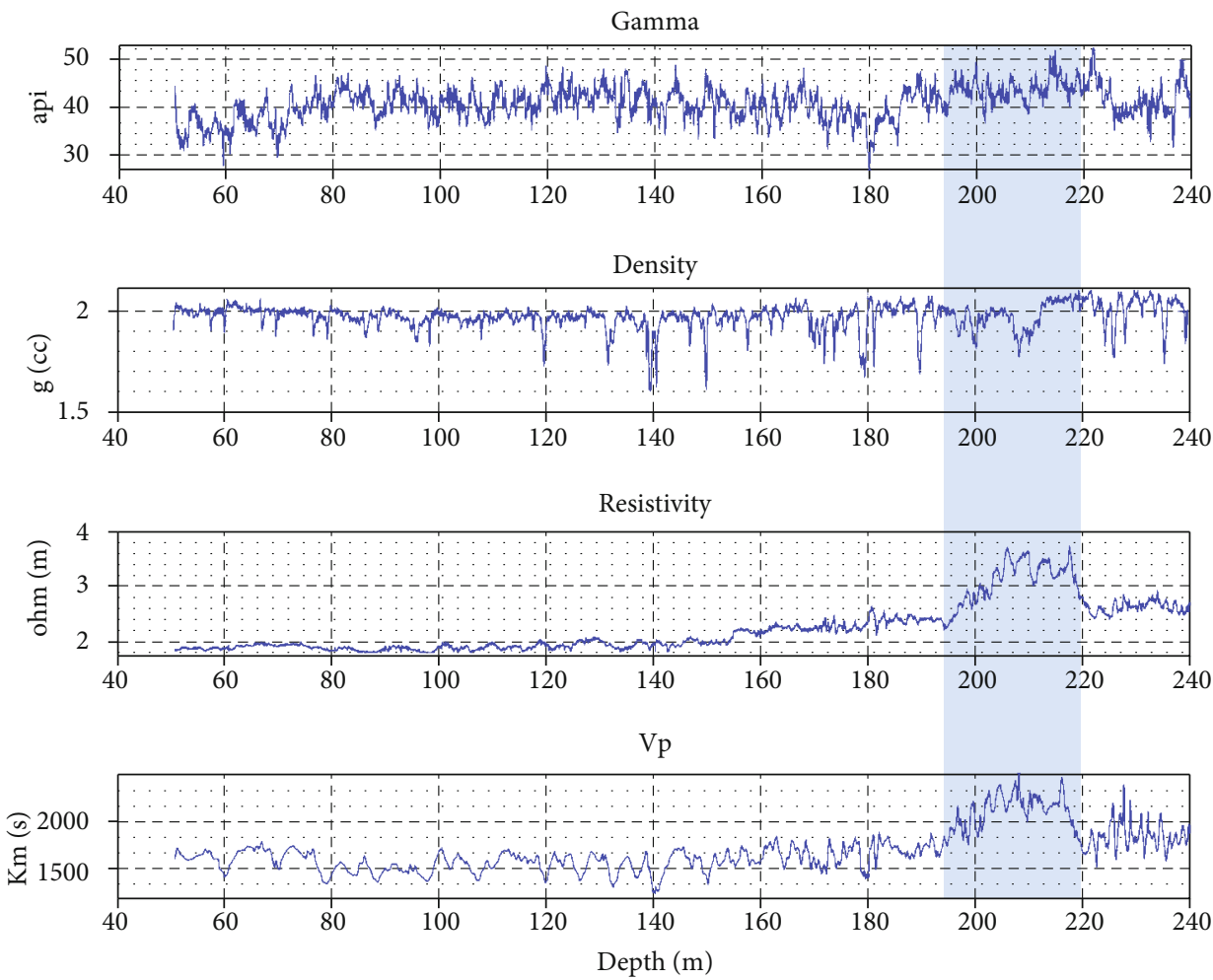

Figure 3: Original curves from well SH2; the blue shaded part indicates the layer of hydrate.
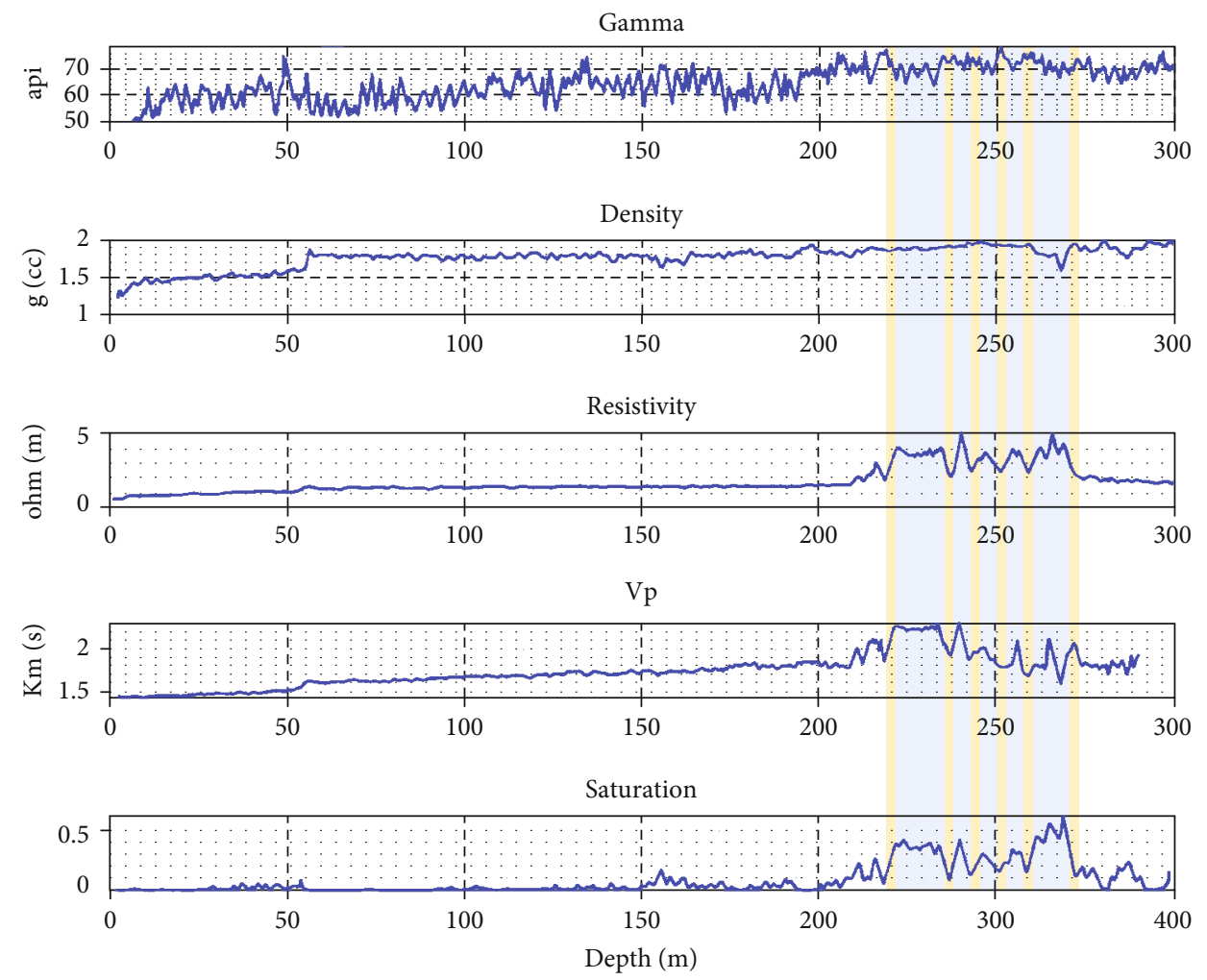

Figure 4: Curves from well SH17; the blue shaded part indicates the layer of hydrate, and the yellow shaded area indicates the nonhydrate layer. 


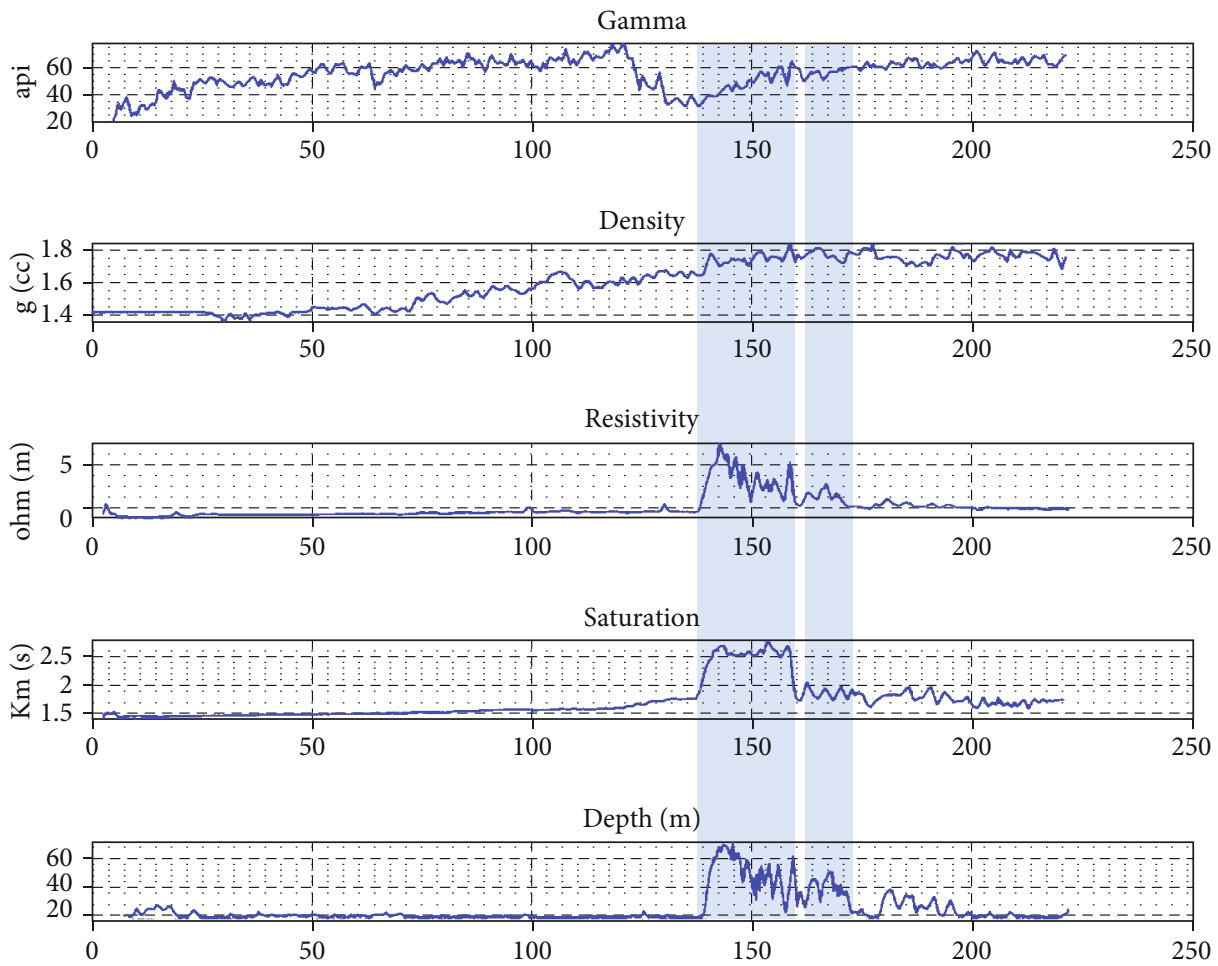

FIgURE 5: Original curves from well SH19; the blue shaded part indicates the layer of hydrate.

provide excellent hydrate development conditions. According to the special structure of the foraminifera shell, it is difficult to describe its characteristics in existing rock physics models with a single cementation mode. Additionally, some scholars have speculated that the hydrate in the pores of the grains occurs within the foraminifera shell, thereby reducing the cementation of the hydrate on the sediment particles [29].

The geothermal gradients estimated based on the in situtemperature measurements at sites W07, W08, and W09 $\left(102-111^{\circ} \mathrm{C} / \mathrm{km}\right)$ are higher than those at the background site $\mathrm{W} 01\left(65^{\circ} \mathrm{C} / \mathrm{km}\right)$. It is inferred that the fractures and faults in the gas chimneys at sites W07, W08, and W09 are the major contributors to the efficient fluid pathways and high geothermal gradients. Therefore, the development of structures such as faults and undercuts in the hydrate reservoirs in the Shenhu area provides conditions for the upward migration of free gas to form stable hydrate distribution areas and simultaneously increases the thermal gradient of the area. The multibeam topographic survey data suggest that cold springs have developed in this area, so the heat flow value of the study area is higher; thus, the structural characteristics of the geology in this area control the temperature conditions [25].

\section{Methodology}

4.1. Composition of the Rock. Based on the above analysis, this paper constructs a unified petrophysical model to address the following problems: (1) the hydrate occurrence state and the reservoir structure in the model must be similar to the patchy distribution in the background stratum, with strong horizontal and vertical heterogeneity; (2) there are diversified cementation modes in the hydrate reservoir in the foraminifera-controlled area; and (3) due to the structural characteristics of the target area, the abnormal heat flow value affects the thermoelastic properties of the study area.

The input parameters, such as the rock matrix modulus and density, are usually not easy to obtain directly and vary among different regions. The input parameters of the same region will also change with the change in depth. The application of empirical parameters will inevitably cause large errors [15]. Therefore, this article determines the matrix composition based on the borehole data of the hydrate in the study area and other geological data, with the density borehole data and Hashin-Shtrikman-Walpole limits as constraints.

$$
E\left(\alpha_{P}\right)=\min \left\|\rho^{M}-\rho^{E}\left(\varnothing, \alpha_{\mathrm{P}}, \rho_{\text {clay }}, \rho_{\text {silt }}, \rho_{\text {sand }} \cdots \cdots\right)\right\| \text {, }
$$

where $\alpha_{\mathrm{P}}$ is the percentage of each component in the rock; $\rho_{\text {clay }}$ is the clay density; $\rho_{\text {silt }}$ is the mud density; $\rho_{\text {sand }}$ is the sandy density; $\rho^{M}$ is the reservoir density; and $\varnothing$ is the porosity. In the pore-filling model, the matrix is a homogeneous mixture of solid minerals such as quartz, calcite, and clay. In the particle cementation model, the matrix contains hydrates in addition to the abovementioned solid minerals. When the details of the geometric distribution of each solid component are unknown, the Hashin-Shtrikman upper and lower limits are the narrowest elastic modulus limits, which 

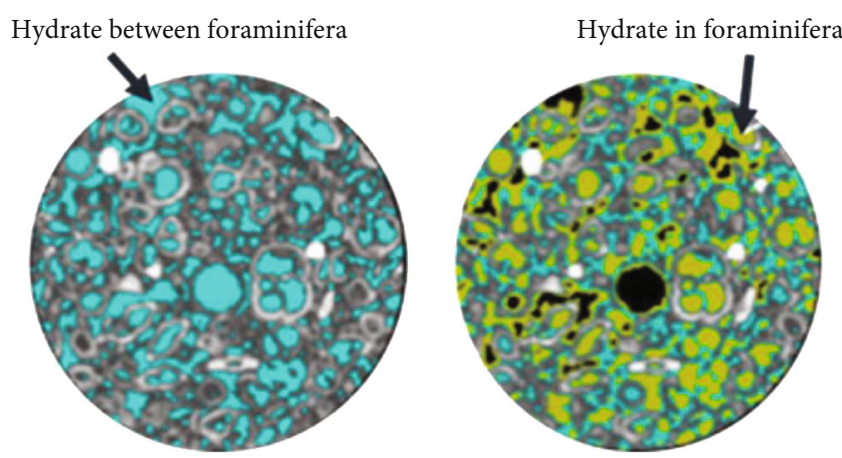

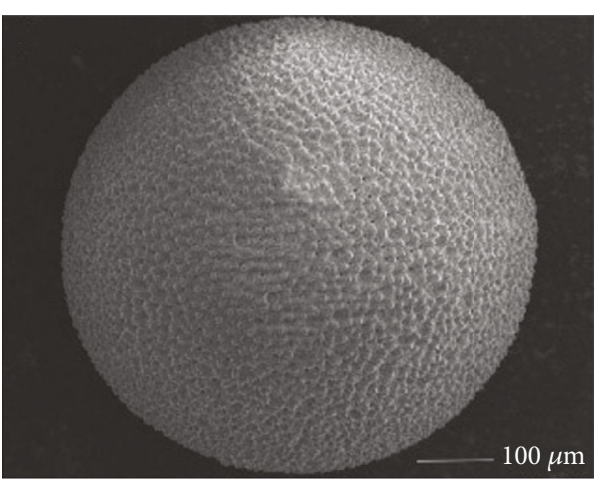

(a)

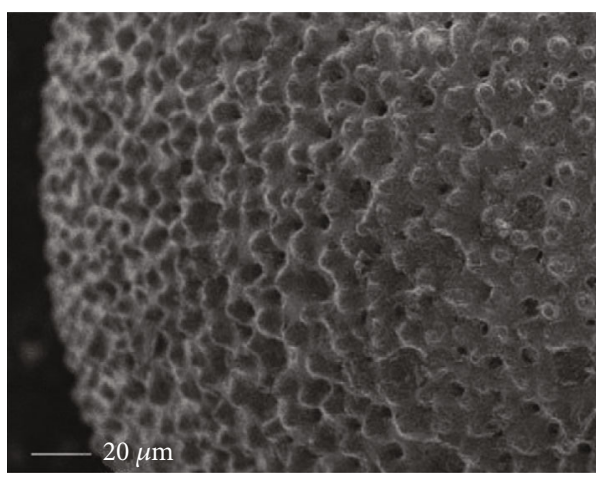

(c)

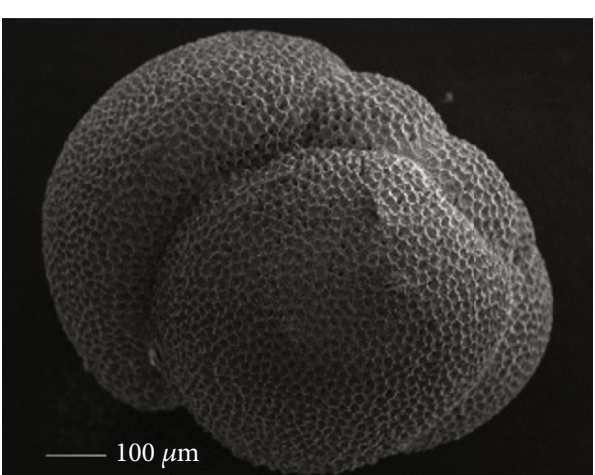

(b)

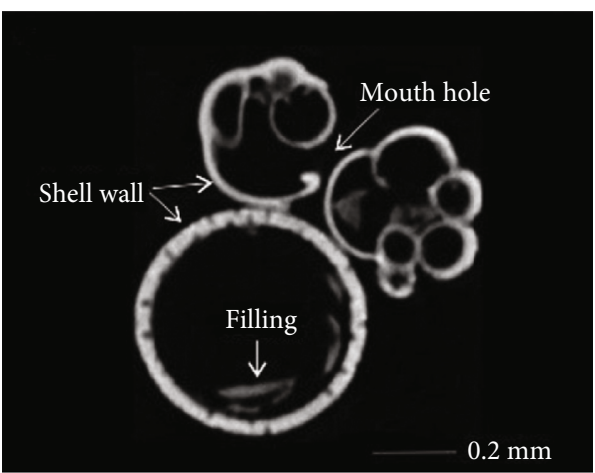

(d)

FIGURE 6: Characteristics of a hydrate reservoir rich in foraminifera and surface and internal images of the foraminifera shells (Li et al., 2016).

are extended to two or more mineral compositions, namely, the Hashin-Shtrikman-Walpole limits [30].

$$
\begin{gathered}
K^{\mathrm{HS} \pm}=\left[\frac{\sum_{i=1}^{n} f_{i}}{\left(K_{i}+4 \mu^{ \pm} / 3\right)}\right]^{-1}-\frac{4 \mu^{ \pm}}{3}, \\
\mu^{\mathrm{HS} \pm}=\left[\sum_{i=1}^{N} \frac{f_{i}}{\left(\mu_{i}+\varepsilon^{ \pm}\right)}\right]^{-1}-\varepsilon^{ \pm} .
\end{gathered}
$$

4.2. Rock Skeleton Construction. The equivalent rock skeleton model is the core part of a rock physics model of a hydrate reservoir, and the structural characteristics of the pore system are the key parameters that affect the elastic properties of the rock skeleton [17]. The DEM model gradually adds pores to the rock matrix in a certain increment.
It utilizes a differential equation that realizes the coupling of the bulk modulus and shear modulus. The coupled differential equivalent equation is decoupled into an ordinary differential equation as follows [3]. Because the calculation process of $P$ and $Q$ is too complicated, a simplified calculation method is given in this article.

$$
\begin{aligned}
& K_{\mathrm{d}}^{*}(\varnothing)=K_{1}(1-\varnothing)^{(P)} e^{\varnothing} \\
& \mu_{\mathrm{d}}^{*}(\varnothing)=\mu_{1}(1-\varnothing)^{(Q)} e^{\varnothing} .
\end{aligned}
$$

Two examples of coefficients $P$ and $Q$ for spherical and nonspherical scatterers are shown in Table 1.

4.3. Saturated Rock Equivalent Model Construction. Pore-filling type: when the medium filling a pore is a nonviscous 


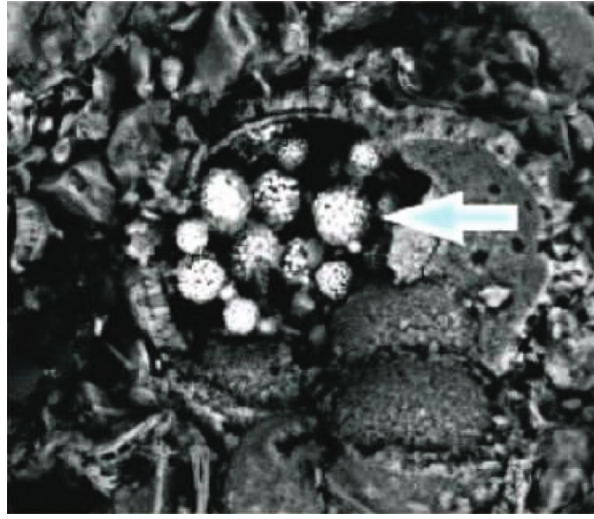

(a)

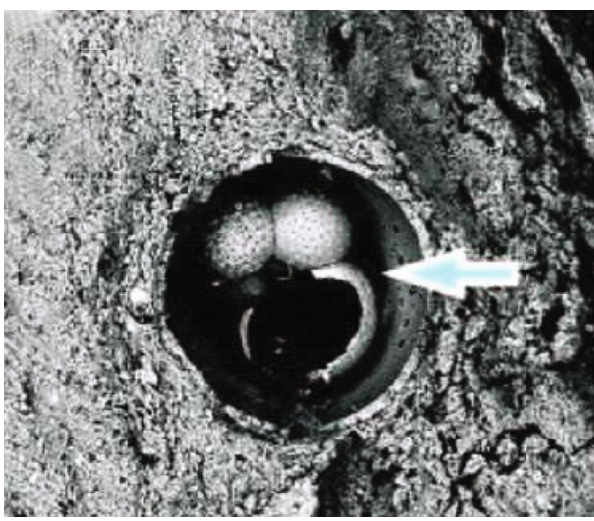

(c)

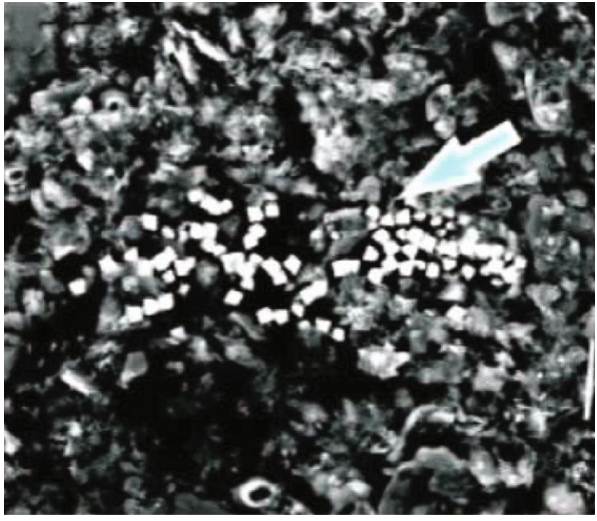

(b)

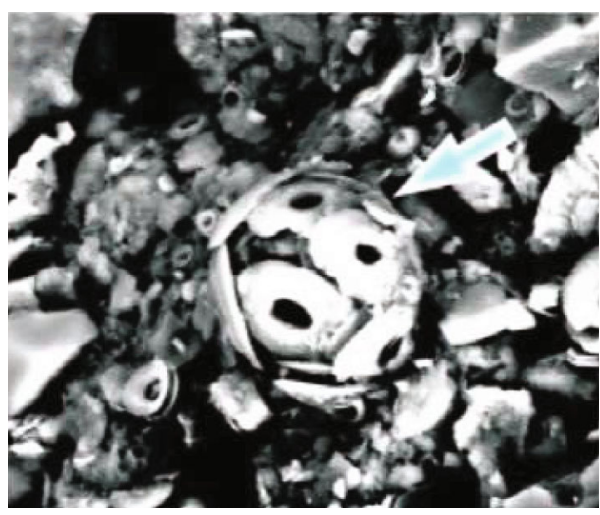

(d)

FIGURE 7: The scanning electron micrograph of W19 well in Shenhu: (a) strawberry pyrite; (b) dispersed particles; (c) foraminifera; (d) coccolith [27].

fluid such as free gas or water, the pore pressure more easily reaches equilibrium, so the bulk modulus can be described by Gassmann's equation. If the filling is viscous hydrate, the pore stress is reduced to the pore pressure [31]. For an isotropic rock skeleton, the elastic modulus can be expressed as follows [32]:

$$
\begin{aligned}
& K_{\mathrm{s}}^{-1}=K_{\mathrm{d}}^{-1}-\left(K_{\mathrm{d}}^{-1}-K_{\mathrm{m}}^{-1}\right)^{2} \times\left[\varnothing\left(K_{i f}^{-1}-K_{\varnothing}^{-1}\right)+\left(K_{\mathrm{d}}^{-1}-K_{\mathrm{m}}^{-1}\right)\right]^{-1}, \\
& \mu_{\mathrm{s}}^{-1}=\mu_{\mathrm{d}}^{-1}-\left(\mu_{\mathrm{d}}^{-1}-\mu_{\mathrm{m}}^{-1}\right)^{2} \times\left[\varnothing\left(\mu_{\mathrm{if}}^{-1}-\mu_{\varnothing}^{-1}\right)+\left(\mu_{\mathrm{d}}^{-1}-\mu_{\mathrm{m}}^{-1}\right)\right]^{-1},
\end{aligned}
$$

where $\mu_{\mathrm{s}}, \mu_{\mathrm{d}}$, and $\mu_{\mathrm{m}}$ are the shear moduli of the pore filling, the dry framework, and the solid matrix, respectively; $K_{\varnothing}$ and $\mu_{\varnothing}$ are the volume and shear moduli related to the pores of the dry framework, respectively; and $K_{\text {if }}$ and $\mu_{\text {if }}$ are the volume and shear modulus of the $i$ type of pore filling.

Particle cementation model: the particle cementation model and the pore-filling model are two extreme models of the hydrate overlay state. In the particle cementation model, solid hydrate directly participates in the formation of the rock skeleton. In the particle cementation model, when the pore filling is a nonviscous fluid such as free gas and water, it does not affect the shear properties of the sediment. The Gassmann equation can be used to calculate the elastic properties of isotropic, fluid-saturated rocks [8].

$$
\begin{aligned}
& K_{\mathrm{s}}=K_{\mathrm{d}}+\alpha^{2} K_{\mathrm{f}}\left[\varnothing+(\alpha-\varnothing) K_{\mathrm{f}} / K_{\mathrm{m}}\right]^{-1}, \\
& \mu_{\mathrm{s}}=\mu_{\mathrm{d}} .
\end{aligned}
$$

In the formula, $\alpha=1-K_{\mathrm{d}} / K_{\mathrm{m}}, K_{\mathrm{s}}, K_{\mathrm{d}}$, and $K_{\mathrm{m}}$ are the bulk moduli of the saturated sediment, dry framework, and solid matrix, respectively, and $\mu_{\mathrm{s}}$ and $\mu_{\mathrm{d}}$ are the shear moduli of the saturated sediment and dry framework, respectively. Gassmann theory suggests that the shear modulus of the saturated sediment is the same as that of the dry skeleton.

Thermoelastic model: there are certain differences in the thermoelastic properties of the natural gas hydrate, free gas, and rock; in particular, the thermal expansion coefficient of free gas is higher than that of natural gas hydrate and rock (Table 2). Elastic waves passing through the hydrate reservoirs and surrounding rock exhibit different thermoelastic responses. The greater the saturation of free gas and hydrate is, the greater the difference, which further affects the elastic parameters of saturated rock. 


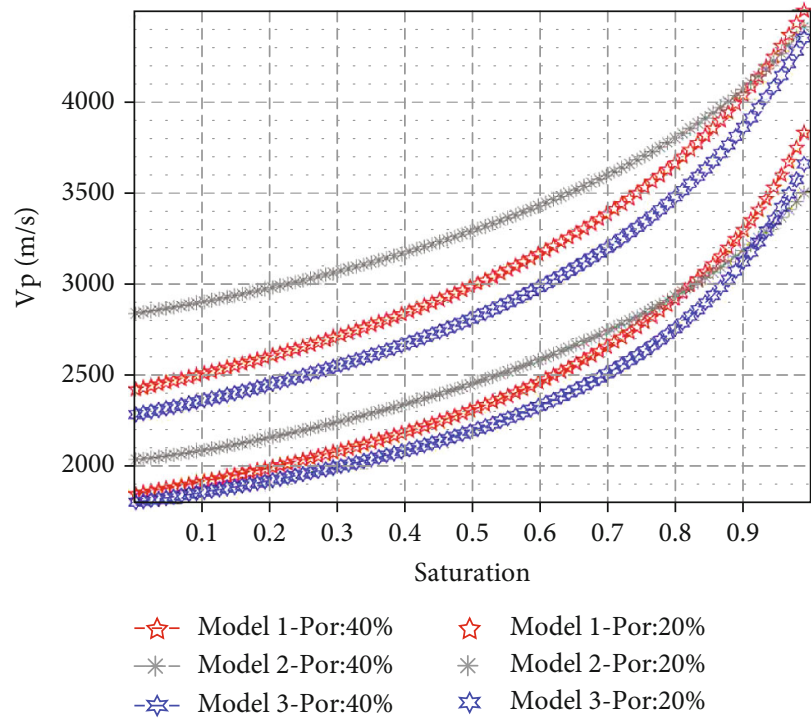

(a)

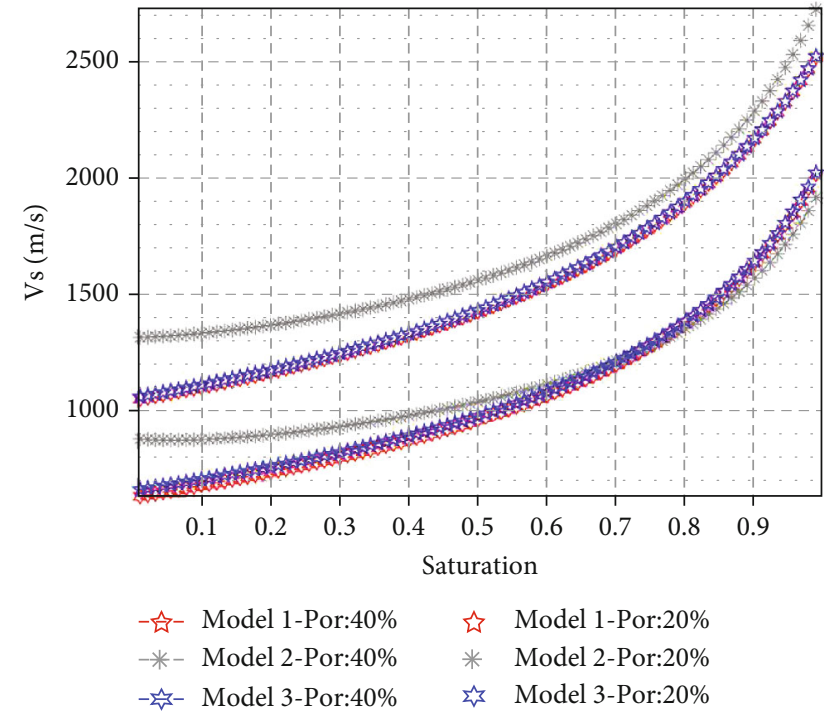

(b)

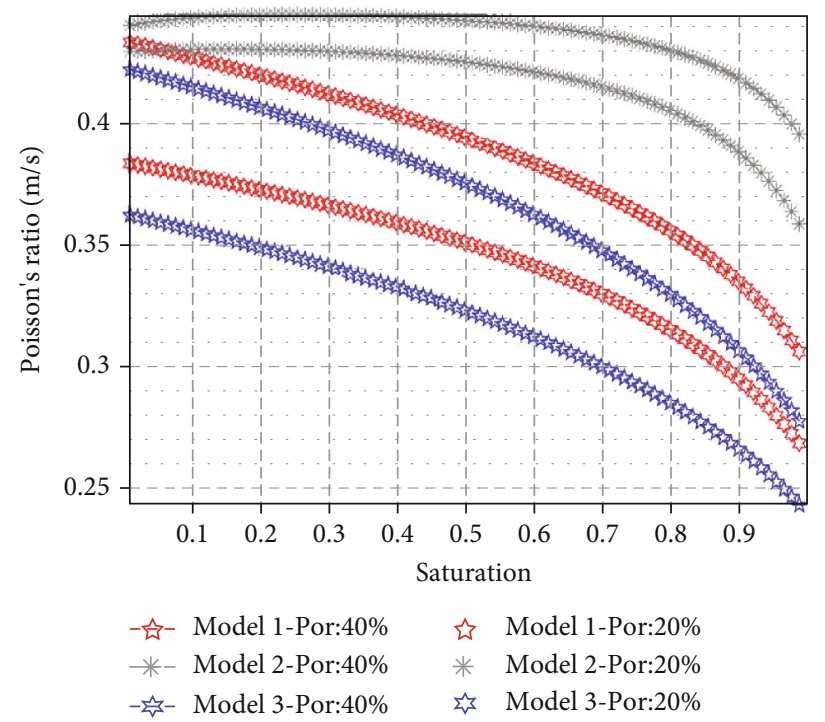

(c)

FIgURE 8: Relationship of $V_{\mathrm{p}}, V_{\mathrm{s}}$, Poisson's ratio, and hydrate saturation of saturated rock in a hydrate reservoir. Model 1 is a pore-filling model, Model 2 is a thermodynamic elastic model, and Model 3 is a particle cementation model; P40 and P20 represent porosities of $40 \%$ and $20 \%$, respectively. (a) Relationship of $V_{\mathrm{p}}$ and hydrate saturation of saturated rock in a hydrate reservoir; (b) relationship of $V_{\mathrm{s}}$ and hydrate saturation of saturated rock in a hydrate reservoir; (c) relationship of Poisson's ratio and hydrate saturation of saturated rock in a hydrate reservoir.

According to the thermoelastic model, the $V_{\mathrm{p}}$ of the deposited layer can be obtained as follows:

$$
\begin{aligned}
V_{\mathrm{p}} & =\left\{\left[\left(K+\frac{4}{3} G\right)+\frac{9 K^{2} \alpha^{2} T_{0}}{\rho_{m} C_{e}}\right] \frac{1}{\rho_{m}}\right\}^{1 / 2} \\
V_{\mathrm{s}} & =\left\{\left[G+r \frac{9 K^{2} \alpha^{2} T_{0}}{\rho_{m} C_{e}}\right] \frac{1}{\rho_{m}}\right\}^{\frac{1}{2}} \\
\alpha & =\left(1-\Phi_{\text {eff }}\right) \alpha_{s}+\Phi_{\text {eff }}\left(S_{w} \alpha_{w}+S_{g} \alpha_{g}\right) \\
C_{e} & =\left(1-\Phi_{\text {eff }}\right) C_{e s}+\Phi_{\text {eff }}\left(S_{w} C_{e w}+S_{g} C_{e g}\right)
\end{aligned}
$$

$$
\begin{aligned}
& \mathrm{K}=\frac{K_{\mathrm{b}}\left(K_{\mathrm{m}}+R\right)}{K_{\mathrm{b}}+R}, \\
& R=\frac{K_{\mathrm{f}}\left(K_{\mathrm{b}}-K_{\mathrm{m}}\right)}{\Phi_{\text {eff }}\left(K_{\mathrm{b}}-K_{\mathrm{f}}\right)},
\end{aligned}
$$

where $\alpha$ is the coefficient of thermal expansion; $C_{e}$ is the specific heat capacity of the material; $9 K^{2} \alpha^{2} T_{0} / \rho_{m} C_{e}$ describes the influence of the thermodynamic properties of the rock on the $V_{\mathrm{p}}$; and $r$ is the coefficient describing the influence of the thermodynamic properties of the rock on the $V_{s}$. 


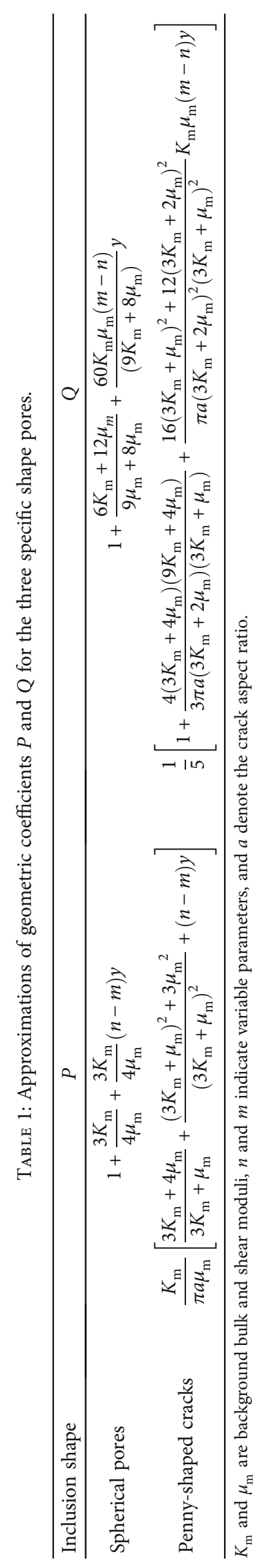


These conditions are applied to the (1) water-saturated layer; (2) water- and hydrate-saturated layer; and (3) water- and free gas-saturated layer.

4.4. Construction of a Heterogeneous Petrophysical Model of a Hydrate Reservoir in the Shenhu Area. According to the analysis of the borehole data, the hydrate reservoir in the area of SH2 contains a large amount of sediment and can be regarded as a sediment-controlled hydrate reservoir, which controls the pore-filling mode. There are many foraminifera observed in the samples from the area of well W19, so the pore-filling mode is mainly controlled by foraminifera. There are also some clay-controlled and sediment-controlled types of reservoirs. Thus, the porefilling model alone cannot accurately describe the complex hydrate distribution state. The hydrate distribution in the area of well W17 is mostly in thin interbedded hydrates, and the patterns of the overlying strata are varied. Therefore, combining the special geological conditions of the Shenhu area and the sensitivity of a hydrate reservoir to the temperature and pressure environment, the thermoelastic effect of
TABle 2: Physical properties of different substances.

\begin{tabular}{lccc}
\hline Substance & $\begin{array}{c}\text { Density } \\
\left(\mathrm{kg} / \mathrm{m}^{3}\right)\end{array}$ & $\begin{array}{c}\text { Specific heat } \\
\text { capacity } \\
(\mathrm{J} /(\mathrm{kg} * \mathrm{~K}))\end{array}$ & $\begin{array}{c}\text { Thermal expansion } \\
\text { coefficient } 10^{-5} * \mathrm{~K}^{-1}\end{array}$ \\
\hline Rock & 2650 & 750 & 0.11 \\
Hydrate & 767 & $2050-2200$ & 10.4 \\
Gas & 88.48 & 2400 & 260 \\
Oil & $790-960$ & 2200 & 22.89 \\
Water & 1095 & 4190 & 20.2 \\
\hline
\end{tabular}

the hydrate reservoir is included in the following analysis. Taking into account the physical properties of the two most representative hydrate cementation modes, pore filling and particle cementation, a petrophysical model with multiple cementation mode characteristics, such as the pore-filling medium, particle cementation mode, and thermoelastic properties, is finally constructed:

$$
\begin{aligned}
& V_{\mathrm{p}(\text { Model } 4)}=\sqrt{\frac{\left(K_{\mathrm{sat} 1} * m_{1}+K_{\mathrm{sat} 2} * m_{2}+(4 / 3)\left(G_{\mathrm{dry} 1} * m_{1}+G_{\mathrm{dry} 2} * m_{2}\right)+\left(9 K^{2} \alpha^{2} T_{0} / \rho_{m} C_{e}\right) * m_{3}\right)}{\rho}}, \\
& V_{\mathrm{s}(\text { Model } 4)}=\sqrt{\frac{\left(G_{\mathrm{dry} 1} * m_{1}+G_{\mathrm{dry} 2} * m_{2}+\left(9 K^{2} \alpha^{2} T_{0} / \rho_{m} C_{e}\right) * m_{3}\right)}{\rho}}
\end{aligned}
$$

where $K_{\text {sat1 }}$ and $G_{\text {dry } 1}$ are the bulk modulus and shear modulus factors of the pore-filling mode, respectively; $K_{\text {sat2 }}$ and $G_{\text {dry2 }}$ are the bulk modulus and shear modulus factors of the particle cementation mode, respectively; $m_{1}$ and $m_{2}$ represent the percentage of pore filling medium and the particle cementation respectively and $9 K^{2} \alpha^{2} T_{0} / \rho_{m} C_{e}$ is the thermoelastic influence factor. In this paper, the particle swarm optimization algorithm is used to obtain the appropriate weights of the parameters $m_{1}, m_{2}$, and $m_{3}$, and the $V_{\mathrm{p}}$ is used as a constraint to obtain a petrophysical model suitable for the hydrate reservoir in the trial mining area in Shenhu. The global optimization model is as follows:

$$
\operatorname{M4}\left(m_{1}, m_{2}, m_{3}\right)=\min \left(\left\|V p-V_{\mathrm{p}(\text { Model } 4)}\right\|^{2}\right),
$$

where $M 4\left(m_{1}, m_{2}, m_{3}\right)$ is the optimal weight matrix; $m_{1}$, $m_{2}$, and $m_{3}$ correspond to the weights of the parameters of the three petrophysical models; and the objective function is $\left\|V p-V_{\mathrm{p}(\text { Model } 4)}\right\|^{2}$. The measured $V_{\mathrm{p}}$ of the borehole is a constraint, and accurate weights of the parameters of the petrophysical model (Model 4) are obtained. In the above formula, the velocity components of the three models (Model 1, Model 2, and Model 3) can be obtained according to the petrophysical model. A simulated annealing algorithm is an optimization model that searches for the objective function in a given model space to achieve the global minimum. This approach has been used in various optimization problems. Therefore, according to the objective function, the simulated annealing method is used to obtain the model coefficients.

(1) The range of estimated parameters of the given model is $0-1$. An initial value $\left(m_{1}, m_{2}, m_{3}\right)$ is randomly selected to calculate the objective function value $E\left(m_{0}\right)$. Then, a new model $m$ is obtained by $m_{0}$ perturbation, and the new objective function value $E(m)$ is obtained as follows: $d E=E(m)-E\left(m_{0}\right)$. If $d E<0$, the new model $m$ is accepted; otherwise, the new model $m$ is accepted at probability $P$

$$
P=\left[\frac{1-(1-h) \Delta E}{T}\right]^{1 /(1-h)}
$$

(2) At each temperature $T$, step (1) is repeated in an iterative process

(3) As the temperature $T$ is slowly decreased, the above steps are repeated until the results meet the 
convergence conditions. The analog disturbance function is given by the following expressions:

$$
\begin{aligned}
& y_{i}=T \operatorname{sgn}(\mu-0.5)\left[\left(\frac{1+1}{T}\right)^{|2 \mu-1|}-1\right], \\
& m_{i}^{\prime}=m_{i}+y_{i}\left(B_{i}-A_{i}\right)
\end{aligned}
$$

Additionally, the cooling method is as follows:

$$
T(K)=T_{0} \alpha^{K^{1 / N}}
$$

where $T_{0}$ is the initial temperature, $K$ is the number of iterations, $N$ is the number of parameters to be inverted, $C$ is a constant, and $0.7 \leq \alpha \leq 1$.

\section{Result}

5.1. Relationship between the Hydrate Cementation Mode, Saturation, and Petrophysical Elastic Parameters. For the $V_{\mathrm{p}}$, the inversion result of the pore-filling model is greater than that of the particle cementation model (Figure 9(a)), but for the $V_{s}$, the inversion result of the particle cementation model is greater than that of the pore-filling model, so the sensitivity of the two models to the elastic modulus is different (Figure 9(b)). With the gradual increase in saturation, the Poisson ratios obtained by the three models show a decreasing trend, and the Poisson ratio corresponding to the pore-filling model is larger than that of the particle cementation model (Figure 9(c)), indicating that the porefilling model exhibits a relatively weaker compression resistance than the particle cementation model. The greater the porosity is, the greater the Poisson ratio, which means that the greater the porosity is, the greater the difference in the vertical deformation and horizontal deformation of the pores.

5.2. Analysis of the Relationship between the Elastic Parameters of the Hydrate-Bearing Rock Formation and Gas Saturation. With the gradual increase in gas saturation, the $V_{\mathrm{s}}$ obtained based on the thermoelastic model changes relatively quickly (Figure 10). Therefore, it is inferred that the thermoelastic model has a strong sensitivity to the shear modulus of the free gas content of hydrate reservoirs, which can be used as an important reference for the evaluation of free gas content in reservoirs. As the free gas saturation increases, the $V_{\mathrm{p}}$ obtained by the pore model becomes greater than that of the particle cementation model. For the $V_{\mathrm{s}}$ among the models tested, the thermoelastic model is the most sensitive to the change in the saturation of free gas, and its corresponding $V_{\mathrm{s}}$ changes greatly. The $V_{\mathrm{p}}$ obtained by the pore-filling model and the particle cementation model exhibit small changes, but the response of these two models to the free gas saturation is also affected by the hydrate saturation. When the hydrate saturation is 0.3 , the $V_{\mathrm{s}}$ obtained by the pore-filling model is much greater than that of the particle cementation model, but as the saturation gradually increases to 0.5 , the $V_{\mathrm{s}}$ value obtained by the particle cementation model exceeds that of the pore-filling model. This result is consistent with Figure 10, which shows that as the hydrate saturation gradually increases, the physical characteristics described by the particle cementation model and the pore-filling model gradually coincide. Theoretically, this result is in line with the actual situation. The changes in Poisson's ratio among the three models are relatively complicated, but the thermoelastic model is the most sensitive to free gas saturation.

5.3. The Relationships between the Pore Aspect Ratio and Rock Physical Elastic Parameters. Figure 11 shows the relationship between the aspect ratio of two different pore structures (spherical pores and penny-shaped cracks) and the $V_{\mathrm{p}}$, $V_{\mathrm{s}}$, and Poisson's ratio of the reservoir when the hydrate saturation is $40 \%$. According to Figure 11, whether it is spherical pores or penny-shaped cracks, the $V_{\mathrm{p}}$ and $V_{\mathrm{s}}$ increase as the pore aspect ratio increases, while the Poisson ratio is inversely proportional. Due to the large variation range of spherical pores, the pore aspect ratio range in this paper is $0-0.5$ (Figures 11(a)-11(c)), while the penny-shaped pore aspect ratio variation range is relatively limited, with a pore aspect ratio range of $0-0.05$ in this paper (Figures $11(\mathrm{~d})$ 11(f)). Comparing the two different pore models, the change of penny-shaped pore aspect ratio is more sensitive to the influence of reservoir $V_{\mathrm{p}}$ and $V_{\mathrm{s}}$ changes. On the contrary, the $V_{\mathrm{p}}$ and $V_{\mathrm{s}}$ which correspond to the spherical pore model are relatively stable.

5.4. Analysis of the Construction Effect of the Heterogeneous Rock Physics Model. According to the method in the third part (Equations (7), (8), and (9)), using the simulated annealing algorithm, the parameters of the heterogeneous reservoir model (Model 4) at the three boreholes (SH2, W17, and W19) are obtained, respectively. The parameter model and accumulated error of $V_{\mathrm{p}}$ are shown in Table 3.

Figure 12 shows a comparison between the $V_{\mathrm{p}}$ and the original borehole curve of the $\mathrm{SH} 2$ well based on the three previously studied models and the newly modified model. The $V_{\mathrm{p}}$ inversion result of Model 1 in the hydrate storage area has a good fit with the $\mathrm{SH} 2$ drilling curve. The velocity of Model 2 outside of the hydrate storage area is slightly lower than that of the $\mathrm{SH} 2$ drilling curve. The velocity of Model 2 in the hydrate occurrence area is similar to the measured curves of Model 1 and the $\mathrm{SH} 2$ data, and the estimated result of Model 3 is higher. According to the vertical and horizontal wave velocities estimated by the modified model, the pore-filling model is more suitable for the hydrate occurrence area, which is consistent with the results of previous studies.

According to the analysis of well W17 (Figure 13), most of the hydrate overburden layers are thin interbeds of hydrate. Comparing the estimation results of $V_{p}$, in the $225-240 \mathrm{~m}$ reservoir section, the estimation results of the pore-filling model are in good agreement with the actual data, and the particle cementation model fits the actual data poorly. The thermoelastic properties of the thin interbed 


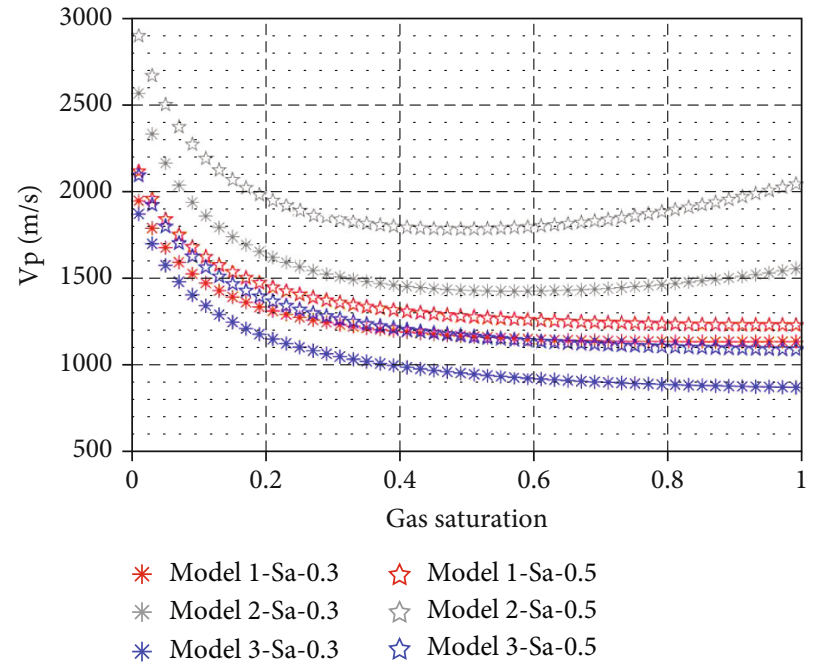

(a)

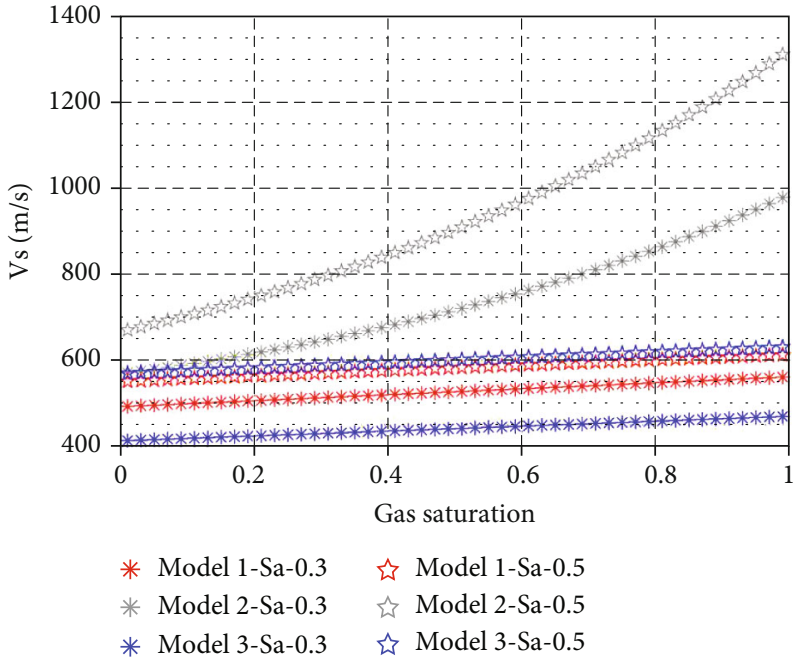

(b)

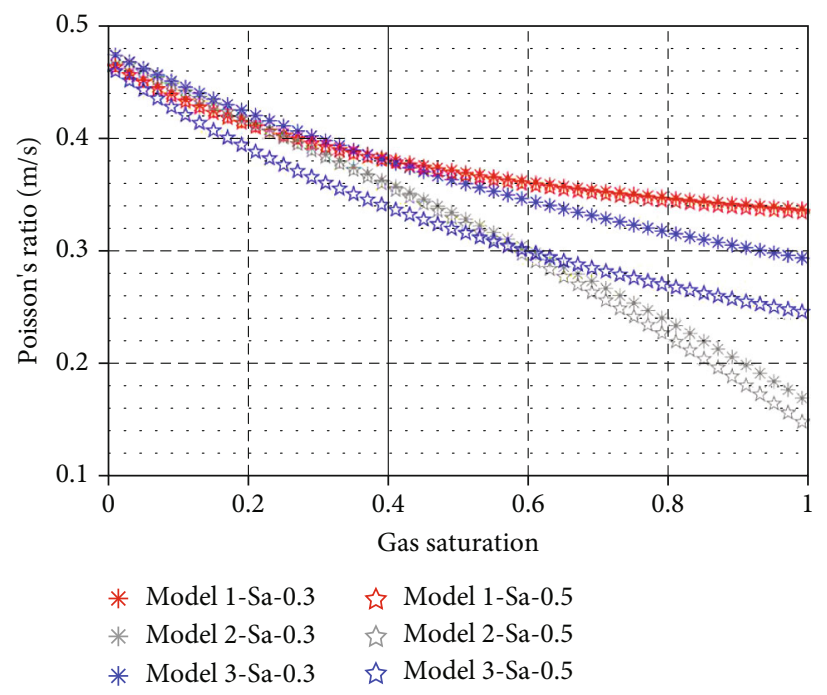

(c)

FIgURE 9: Variations in the elastic parameters of a hydrate-bearing rock formation with gas saturation. Model 1 is a pore-filling model, Model 2 is a thermodynamic elastic model, and Model 3 is a particle cementation model. (a) Relationship between $V_{\mathrm{p}}$ and gas saturation; (b) relationship between $V_{s}$ and gas saturation; (c) relationship between Poisson's ratio and gas saturation.

area $(240-280 \mathrm{~m})$ of the reservoir are stronger. This article infers that the thin hydrate layer has poor stability and exists in a multiphase state. Free gas and fluid distributions have led to significant thermoelastic effects in this area.

According to the analysis of the W19 drilling curve (Figure 13), in the nonhydrate overburden, the $V_{\mathrm{p}}$ obtained by the three petrophysical models differ slightly from the actual drilling curve. In the overlying foraminiferal sediment layer, the $V_{\mathrm{p}}$ estimated by a single model differs greatly from the actual data measured in the borehole, the estimated result of the particle cementation model is greater than the actual data, and the estimated result of the pore-filling model is less than the actual data. Based on these findings, this paper proposes two hypotheses. First, the existence of foraminifera results in hydrate reservoirs with two or more cementation modes: particle cementation and pore filling.
Second, the particle cementation model in the study area has a better degree of cementation, and its sensitivity to the elastic modulus of the rock is greater than that of the pore-filling model. In addition, the strata underlying the foraminiferal overburden are alternating quartz-controlled and clay-controlled layers [9], and the thermoelastic model of this rock is highly sensitive to the speed of the longitudinal and vertical waves, so the thermoelastic properties of the reservoir are more obvious in this area.

\section{Discussion}

6.1. The Influence of Foraminifera on Petrophysical Models. Foraminifera have a special pore structure due to their shells, and their grain size reaches the fine sand level, and it not only increases the content of the coarse components in a 


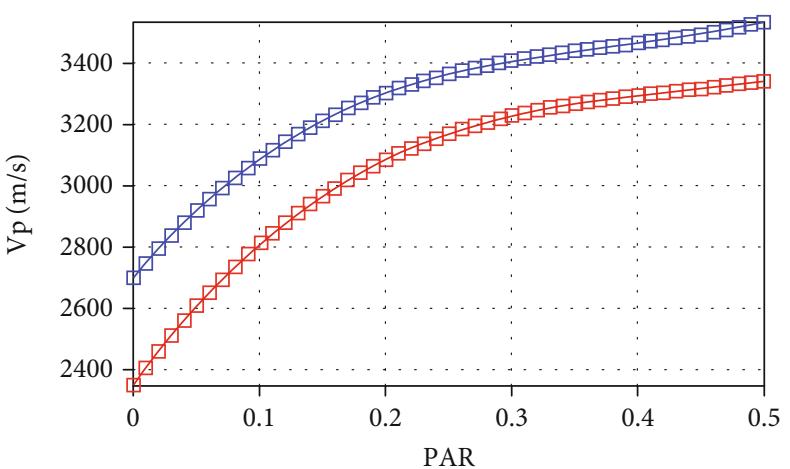

$\square$ Model 1

(a)

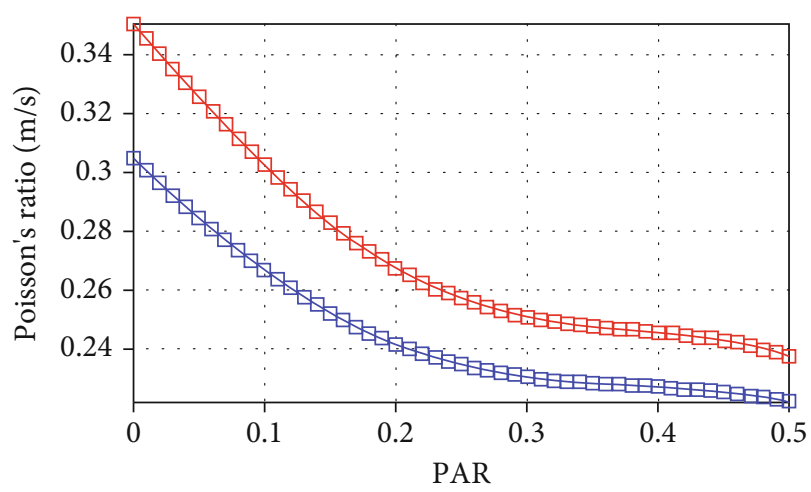

$\square$ Model 1

$\sqsubset$ Model 3

(c)

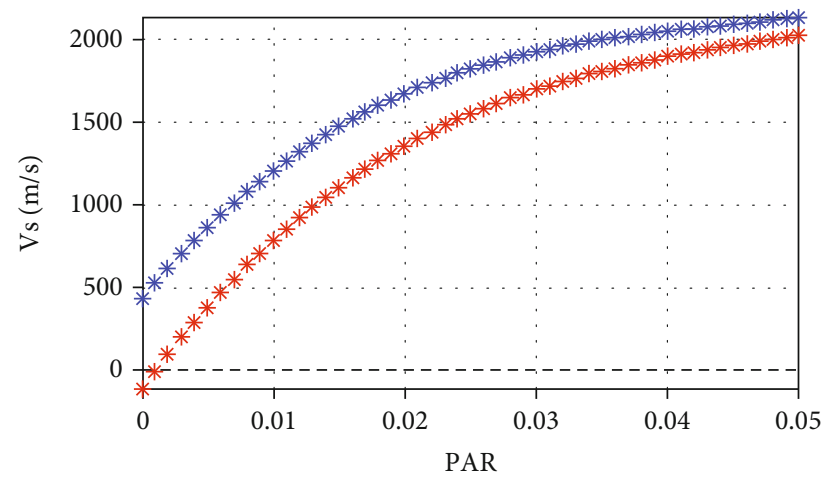

* Model 1

* Model 3

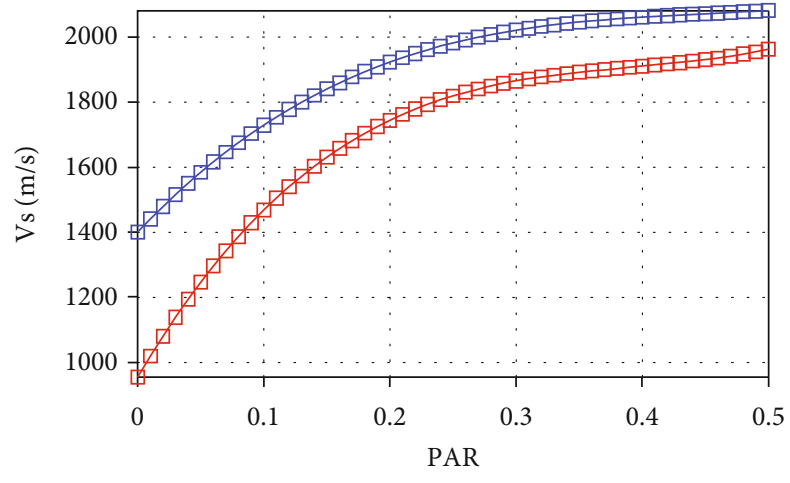

$\square$ Model 1

$\square$ Model 3

(b)

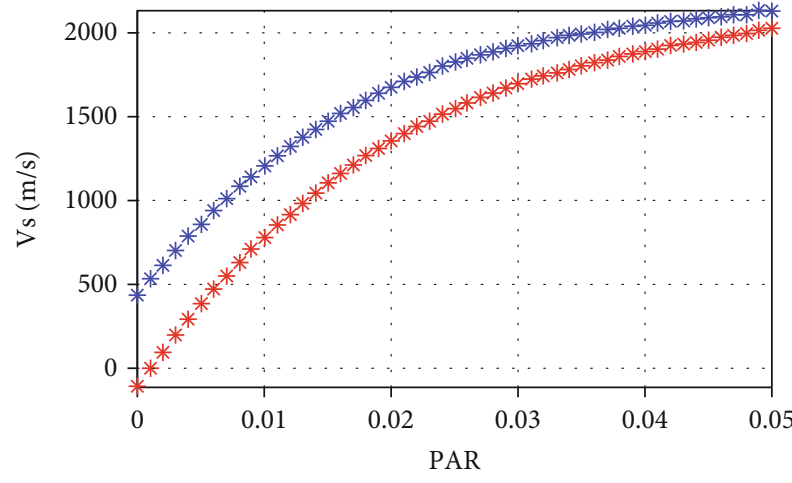

* Model 1

* Model 3

(d)

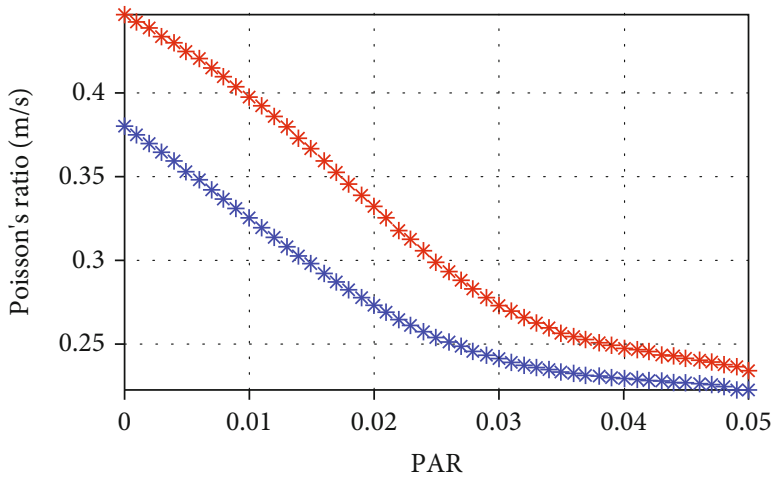

* Model 1

* Model 3

(e)

(f)

FIGURE 10: The relationships between the pore aspect ratio and rock physical elastic parameters; Model 1 is a pore-filling model, and Model 3 is a particle cementation model. (a) Relationship between $V_{\mathrm{p}}$ and PAR of spherical pores; (b) relationship between $V_{\mathrm{s}}$ and PAR of spherical pores; (c) relationship between Poisson's ratio and PAR of spherical pores; (d) relationship between $V_{\mathrm{p}}$ and PAR of pennyshaped cracks; (e) relationship between $V_{s}$ and PAR of penny-shaped cracks; (f) relationship between Poisson's ratio and PAR of pennyshaped cracks.

reservoir but also increases the porosity of the sediment, providing adequate pore space for the growth and storage of hydrate [18]. The pores of the sediments developed by foraminifera are divided into two types: intergranular pores and intragranular pores, both of which can provide a good environment for the growth of hydrates. However, the hydrates in these two pore types have different cementation methods. The intragranular pores can be represented with a 


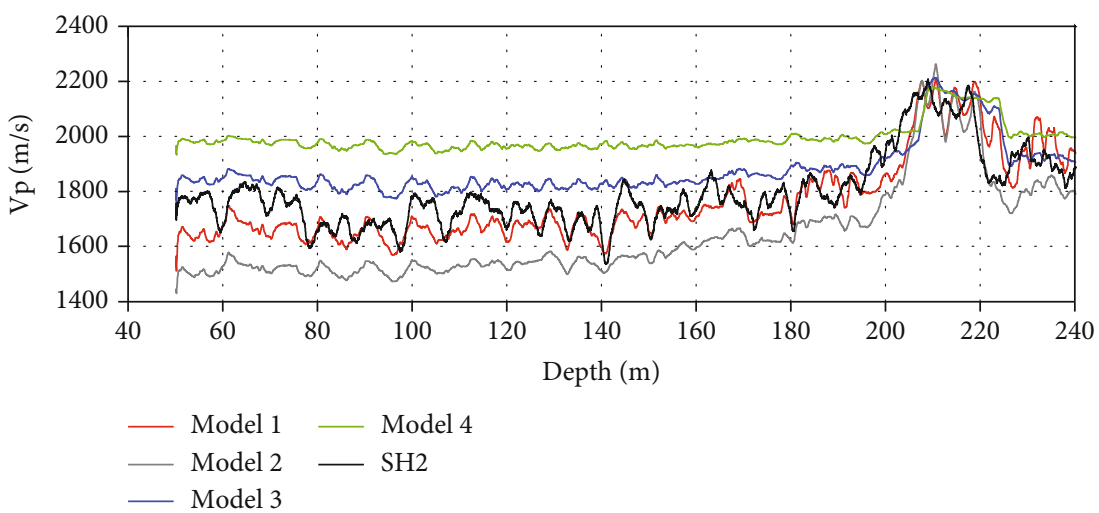

(a)

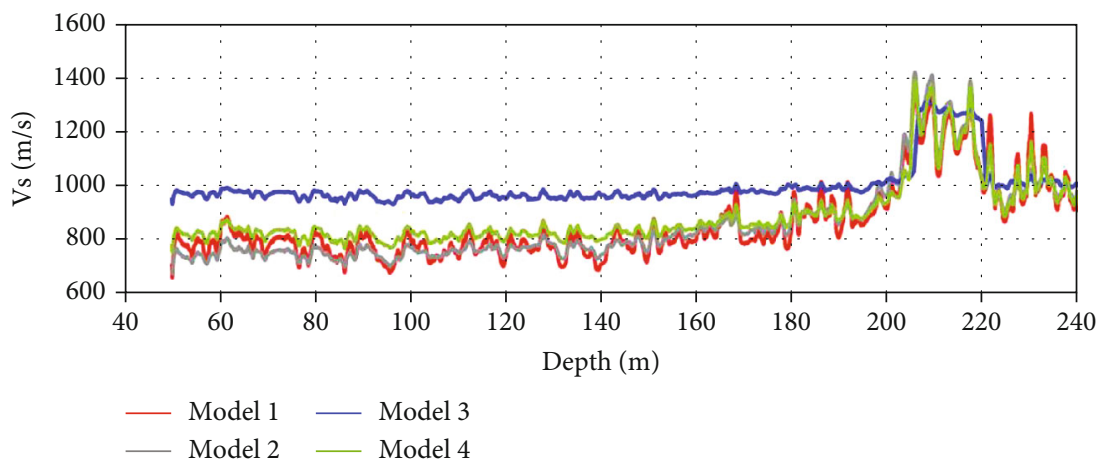

(b)

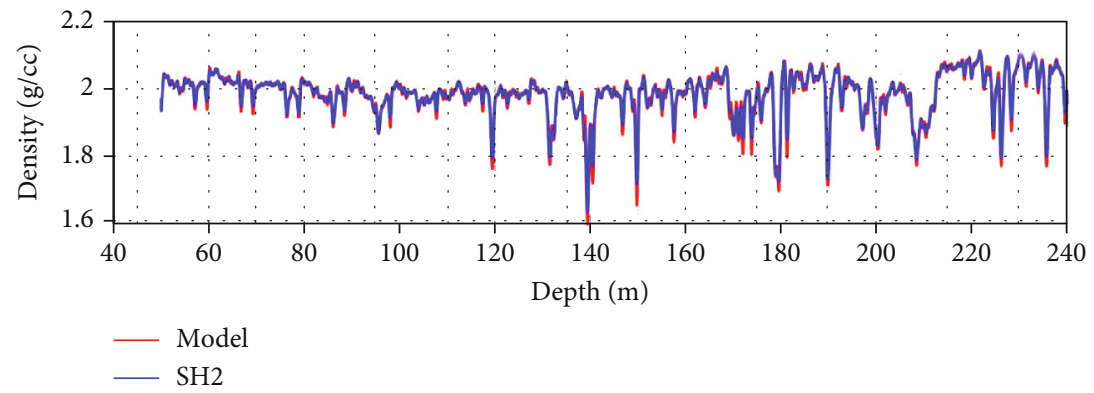

(c)

Figure 11: Comparison of $V_{\mathrm{p}}$ and $V_{\mathrm{s}}$ from site SH2 based on different models (original boreholes): (a) $V_{\mathrm{p}}$ of different models, (b) $V_{\mathrm{s}}$ of different models, and (c) density of model and SH2. Model 1 is a pore-filling model, Model 2 is a thermodynamic elastic model, Model 3 is a particle cementation model, SH2 is a measured drilling curve, and Model 4 is a modified model; SH2 is a measured drilling curve, and Model is the estimated results.

TABLE 3: The heterogeneous reservoir model parameters at the three boreholes (SH2, W17, and W19).

\begin{tabular}{lcccc}
\hline Parameters & $\left|m_{1}\right|$ & $\left|m_{2}\right|$ & $\left|m_{3}\right|$ & $V_{\mathrm{p}}$ deviation \\
\hline SH2 & 0.04 & 0.75 & 0.08 & $20 \%$ \\
W17 & 0.51 & 0.32 & 0.70 & $21 \%$ \\
W19 & 0.74 & 0.43 & 0.12 & $28 \%$ \\
\hline
\end{tabular}

pore-filling model, and the intergranular pores can be represented with a particle cementation model. According to the analysis of the experimental results, the intergranular pore hydrate has a greater influence on the elastic modulus of the sediment, which may be due to the influence of the fora- minifera pore structure, and the hydrate in the intragranular pores has relatively weakened the support of the rock skeleton. Therefore, the pore-filling model alone cannot accurately describe the petrophysical parameters of hydrates in drilling areas with high foraminifera contents. According to the model weight parameters in well 19, the weights of the pore-filling (0.43) and particle cementation models (0.74) are relatively large and equivalent; it is consistent with the type of hydrate covering caused by the presence of foraminifera.

6.2. The Influence of Thermoelastic Characteristic on Petrophysical Models. The temperature and pressure conditions required for the generation of hydrate resources 


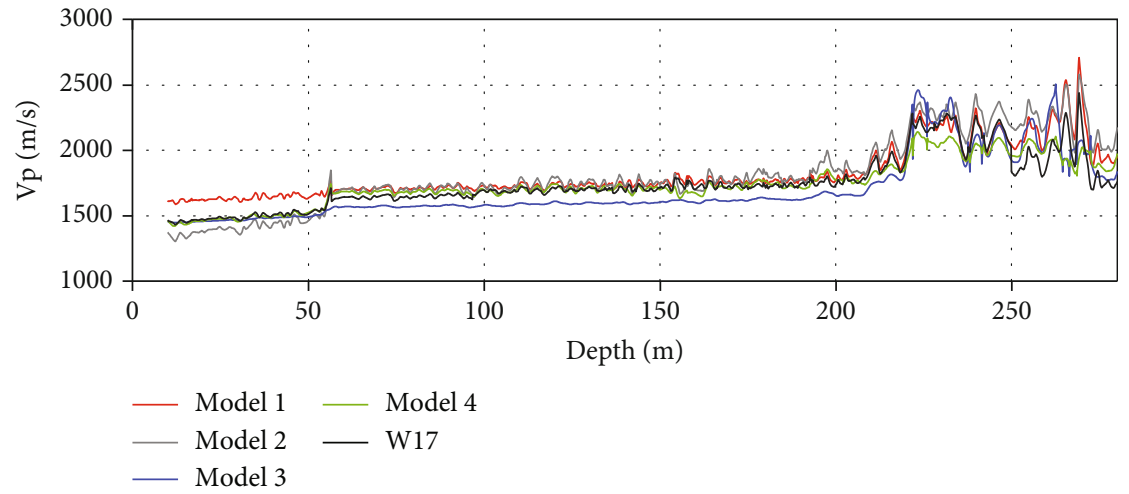

(a)

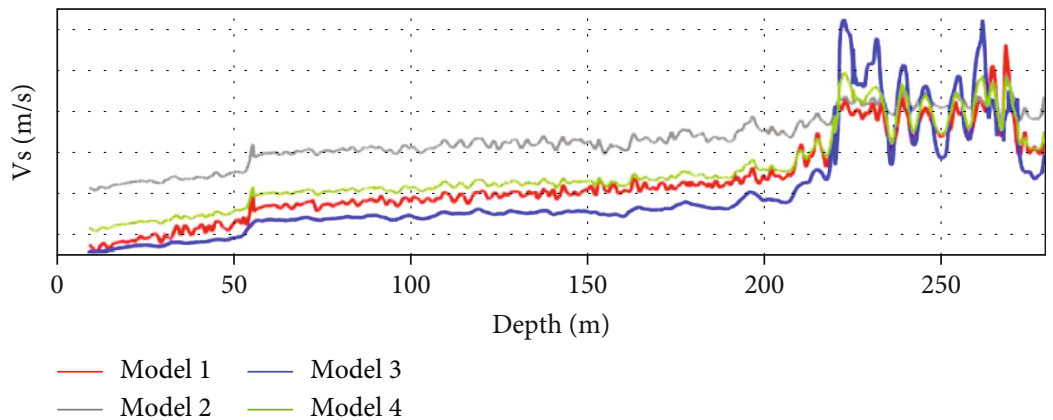

(b)

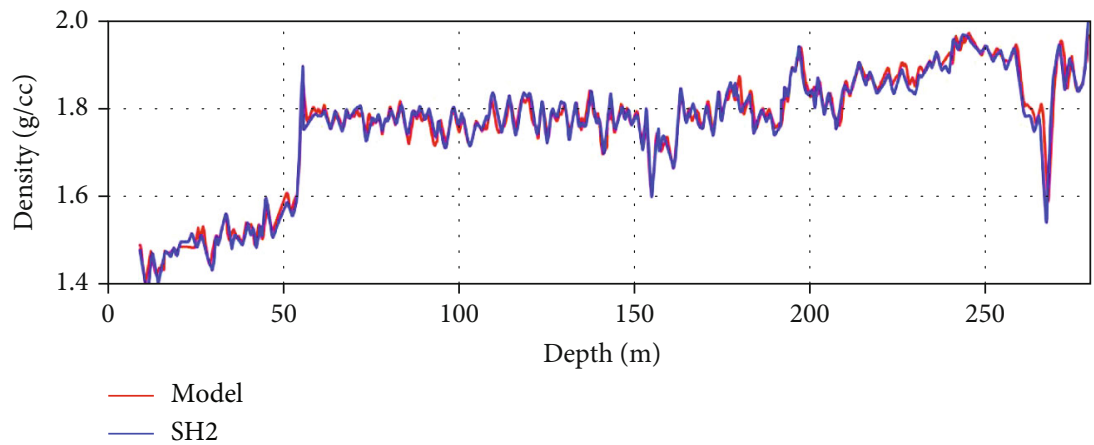

(c)

Figure 12: Comparison of $V_{\mathrm{p}}$ and $V_{\mathrm{s}}$ at well W17 based on different models (original boreholes): (a) $V_{\mathrm{p}}$ of different models, (b) $V_{\mathrm{s}}$ of different models, and (c) density of Model and SH2. Model 1 is a pore-filling model, Model 2 is a thermodynamic elastic model, Model 3 is a particle cementation model, SH2 is a measured drilling curve, and Model 4 is a modified model; data 1 is a measured drilling curve, and data 2 is the estimated results.

determine the physical properties of the hydrate reservoir rocks. Previous studies have shown that the gas sources of hydrate reservoirs in the Shenhu area are mostly biogas and thermogenic gas. Diapirs and faults provide pathways for the upward transportation of deep thermogenic gas and the formation of hydrates in shallow layers and thus simultaneously increase the heat flow value of the shallow layer [33]. Through the analysis of the weight parameters of the petrophysical model of well 17 , the thermoelastic characteristic parameters (0.7) are relatively large, so this is consistent with the existence of free gas and hydrate interactive layers in well 17. Thus, the thermoelastic properties of gas are obviously different from those of other framework materials. In the area of thin interbedded hydrates (W17), due to the sen- sitivity of hydrates to temperature and pressure conditions, the phase state of hydrates is unstable, so there is a greater probability of the existence of fluid media such as free gas, so the influence of its thermoelastic properties cannot be ignored.

The weights of the parameters can be changed in the study of the petrophysical characteristics of the correction model in different drilling areas or different research areas. According to the weight parameters obtained from the three borehole positions, the state of hydrate coverage near the borehole can be inferred. For example, for the three borehole locations, the pore-filling model has the largest weight, which further proves that most of the hydrate-covering models in the Shenhu waters are pore-filling types. The 


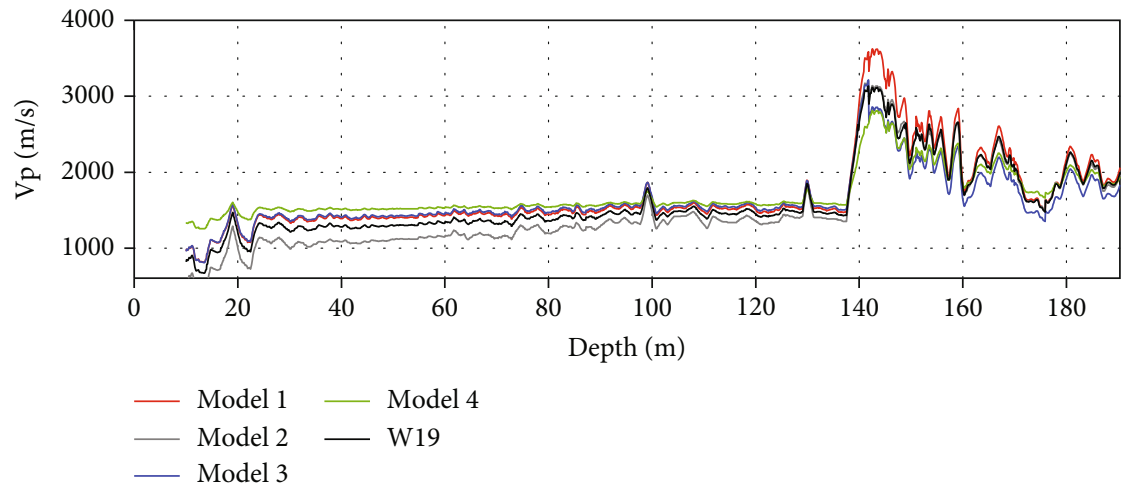

(a)

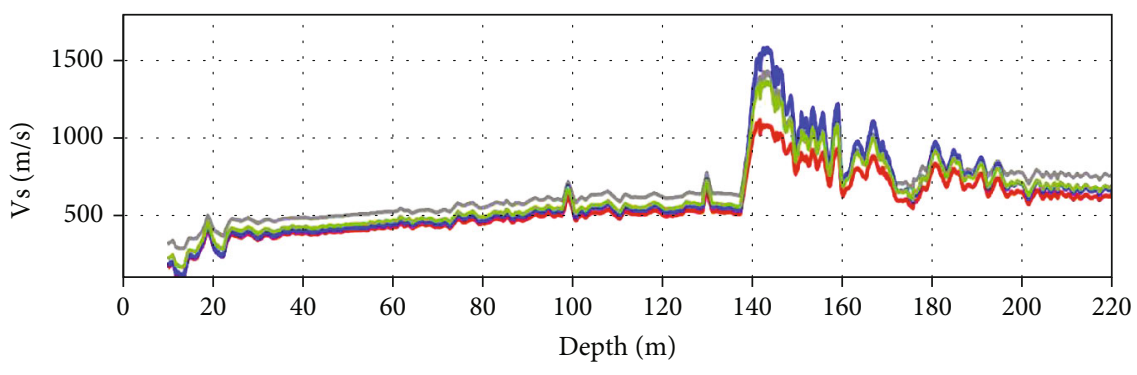

- Model 1 Model 3

- Model $2 \longrightarrow$ Model 4

(b)

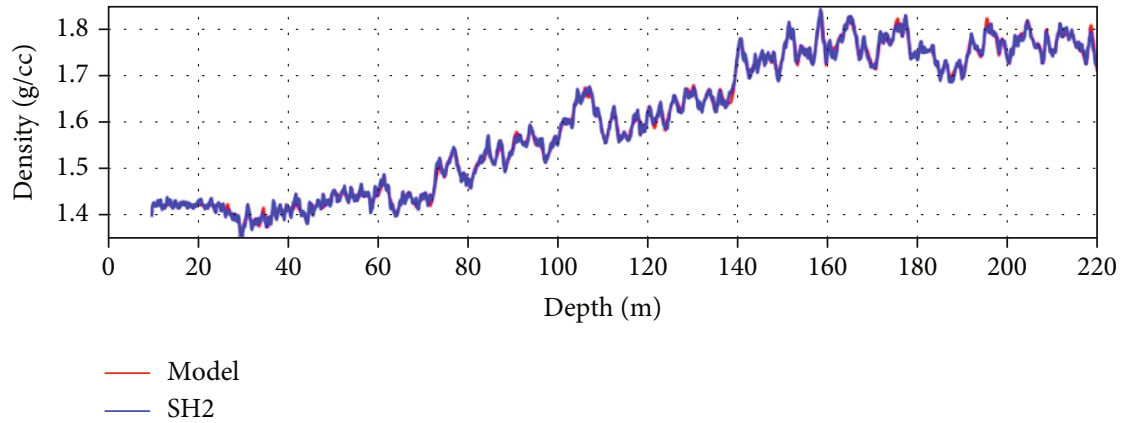

(c)

Figure 13: Comparison of $V_{\mathrm{p}}$ and $V_{\mathrm{s}}$ at well W19 based on different models (original boreholes): (a) $V_{\mathrm{p}}$ of different models, (b) $V_{\mathrm{s}}$ of different models, and (c) density of Model and SH2. Model 1 is a pore-filling model, Model 2 is a thermodynamic elastic model, Model 3 is a particle cementation model, SH2 is a measured drilling curve, and Model 4 is a modified model; data 1 is a measured drilling curve, and data 2 is the estimated results.

weight of the thermoelastic model of well 17 is relatively large, indicating that its thermoelastic characteristics are more obvious than those of the other two boreholes. This new model overcomes the limitation of using a single petrophysical model to describe the petrophysical characteristics of complex sea areas under the influence of multiple factors.

\section{Conclusion}

According to the log curve analysis of the study area, the structure and cementation modes of hydrate reservoirs at different well positions are quite different. It is difficult for a petrophysical model with the same characteristic parameters to achieve a qualitative description of the petrophysical characteristics of the entire target area. Utilizing multiple petrophysical models to describe regional characteristics is also not conducive to the quantitative inversion of physical parameters. Therefore, the construction of an adaptable petrophysical model that is applicable in an entire target area is the key to characterizing hydrate reservoirs through seismic data analysis.

Combining the structural geology of the Shenhu area and the unique temperature and pressure conditions of hydrate reservoirs, the effect of heat flow on the petrophysical elastic parameters of hydrate reservoirs is considered. To improve the accuracy of hydrate reservoir descriptions based on the physical properties of hydrate reservoir rocks rich in foraminifera, a petrophysical model that takes into account 
the existence of multiple cementation modes is constructed. The characteristic parameters of the three models are all included in the correction model.

Identifying the microscopic characteristics of hydrate reservoirs and establishing quantitative relationships among the microstructure, physical properties and elastic properties are important links in realizing the quantitative detection of hydrates. The hydrate reservoirs in the Shenhu area are mostly multiphase reservoirs, with solid, liquid, and gas components. Petrophysical parameters, such as the bulk modulus, the shear modulus, and Poisson's ratio, are important parameters for hydrate reservoir phase identification.

\section{Data Availability}

In this paper, well SH2, well W17, and well W19 are selected as the research objects, and the drilling positions are indicated in Figure 2. The borehole data were obtained in 2007 and 2015, the data sampling interval was $0.02 \mathrm{~m}$, and the borehole depth was $200-300 \mathrm{~m}$. In addition, the seismic data considered in this work are from a seismic section closest to station $\mathrm{SH} 2$. According to the BSR response, the location of the hydrate distribution areas and the structure of the surrounding rock can be clearly seen. The seismic data features and acquisition methods are as follows: on June 7-14, 2015, a stereoscopic detection campaign was carried out in the Shenhu area with Struggle No. 4 and the Treasure Ship (equipped with single-source, single-cable marine horizontal streamers, OBSs, and VCs) by the Guangzhou Marine Geological Survey. A total of 11 OBS stations and 2 VC sets were installed, and 24 lines of multichannel seismic surveys were completed over a total distance of $369.7 \mathrm{~km}$. The layout of the work area is shown in Figure 2. For the horizontal streamers, a unilateral receiving observation system was adopted with a single-sided cannon and 360 channels, and the fold number was 45 . The borehole data and seismic data used in this article belong to commercial data and can be used for scientific research, but it is not convenient for disclosure. If the reader wants to use the drilling data for analysis, the drilling data can be digitized according to the published literature, or contact me by email. Regarding the stereo detection data, we are sorry that it cannot be shared due to copyright reasons. I apologize and thank you from the bottom of my heart!

\section{Conflicts of Interest}

The authors declare that they have no conflicts of interest.

\section{Acknowledgments}

The study is supported by the National Natural Science Foundation of China (Nos. 91858215, 91958206, 42106070, 41304096, and 41876053), the National Key Research and Development Plan (2018YFC1405901 and 2017YFC0307 401 ), and the National Science and Technology Major Project of China (2016ZX05024-001-002).

\section{References}

[1] C. L. Liu, Y. G. Ye, and Q. G. Meng, "The characteristics of gas hydrates recovered from Shenhu area in the South China Sea," Marine Geology, vol. 307-310, no. 3, pp. 22-27, 2012.

[2] S. X. Yang, M. Zhang, and J. Q. Liang, "Preliminary results of China's third gas hydrate drilling expedition: a critical step from discovery to development in the South China Sea," Fire in the Ice, vol. 15, no. 2, pp. 1-21, 2015.

[3] X. Y. Yin and X. X. Liu, "Research status and progress of the seismic rock-physics modeling methods," Geophysical Prospecting for Petroleum, vol. 55, no. 3, pp. 309-325, 2016.

[4] X. Q. Liu, H. S. Liu, L. Xing, Y. X. Yin, and J. H. Wang, "Seismic low-frequency shallow beneath gas hydrate in the Shenhu based on the stereoscopic observation system," Journal of Earth Science, vol. 29, no. 3, pp. 669-678, 2018.

[5] A. Reuss, "Calculation of the flow limits of mixed crystals on the basis of the plasticity of monocrystals," Zeitschift fur Angewandte Mathematik, vol. 9, pp. 49-58, 1929.

[6] W. Voigt, Lehrbuch der kirstallphysik, Leipzig: Teubner, Advances in Earth Science, 1928.

[7] S. Y. Fu and J. A. Lu, "The characteristics and origin of gas hydrate in Shenhu area, South China Sea," Marine Geology Letters, vol. 26, no. 9, pp. 6-10, 2010.

[8] F. Gassmann, "Uber die Elastizitat poroser Medien, Veirteljahrsschrift der Naturforschenden Gesellschaft in Zurich," Chinese Journal of Geophysic, vol. 96, pp. 1-23, 1951.

[9] J. Qian, X. Wang, T. S. Collett, Y. Guo, D. Kang, and J. Jin, "Downhole log evidence for the coexistence of structure II gas hydrate and free gas below the bottom simulating reflector in the South China Sea," Marine and Petroleum Geology, vol. 98, pp. 662-674, 2018.

[10] U. Shankar and M. Riedel, "Gas hydrate saturation in the Krishna-Godavari basin from P-wave velocity and electrical resistivity logs," Marine and Petroleum Geology, vol. 28, pp. 1768-1778, 2011.

[11] M. B. Helgerud, J. Dvorkin, A. Nur, A. Sakai, and T. Collett, "Elastic-wave velocity in marine sediments with gas hydrates: effective medium modeling: geophysics," Research Letters, vol. 26, pp. 2021-2024, 1999.

[12] M. W. Lee and T. S. Collett, "Unique problems associated with seismic analysis of partially gas-saturated unconsolidated sediments," Marine \& Petroleum Geology, vol. 26, no. 6, pp. 775$781,2009$.

[13] J. L. Wang, J. Priyank, H. Seth, H. Patrick, and S. G. Wu, "Gas hydrate quantification using full-waveform inversion of sparse ocean-bottom seismic data: a case study from Green Canyon Block 955, Gulf of Mexico," Geophysics, vol. 83, no. 4, pp. 167-181, 2018.

[14] W. Wei, J. H. Zhang, R. Z. Yue et al., "A review of research and development hotspots of natural gas hydrates in 2017," Science and Technology Review, vol. 36, no. 1, pp. 83-90, 2017.

[15] W. F. Waite, J. C. Santamarina, D. D. Cortes et al., "Physical properties of hydrate-bearing sediments," Reviews of Geophysics, vol. 47, pp. 465-484, 2009.

[16] X. Q. Liu, H. S. Liu, and L. Xing, "Estimation of S-wave velocity for gas hydrate reservoir in the Shenhu area, north South China Sea," Journal of Ocean University of China, vol. 17, no. 5, pp. 1091-1102, 2018.

[17] X. J. Wang, J. Qian, T. S. Collett et al., "Characterization of gas hydrate distribution using conventional 3D seismic data in the 
Pearl River Mouth Basin, South China Sea," Interpretation, vol. 4, no. 1, pp. SA25-SA37, 2016.

[18] C. F. Li, G. W. Hu, W. Zhang et al., "Effects of foraminifera on the formation and occurrence of natural gas hydrate in finegrained sediments in the Shenhu area of the South China Sea," Science China: Earth Sciences, vol. 46, no. 9, p. 1223, 2016.

[19] S. X. Yang, J. Q. Liang, and J. G. Lu, "New understandings on the characteristics and controlling factors of gas hydrate reservoirs in the Shenhu area on the northern slope of the South China Sea," Earth Science Frontiers, vol. 24, no. 2, pp. 1-14, 2017.

[20] J. Liang, M. J. Wang, and H. B. Wang, "Relationship between the sonic logging velocity and saturation of gas hydrate in Shenhu area, northern slope of South China Sea," Geoscience, vol. 23, no. 2, pp. 217-223, 2009.

[21] Z. Q. Wu, S. G. Wu, S. Y. Tong, H. S. Liu, and Y. B. Zhang, "Research on seismic acquisition technology based on marine oil and gas exploration in the South Yellow Sea," Chinese Journal of Geophysics, vol. 54, no. 4, pp. 1061-1070, 2011.

[22] X. W. Luan, Y. S. Qin, and X. H. Zhang, "The stability of gas hydrate in the slope of East China Sea and neighboring trough basin area," Chinese Journal of Geophysics, vol. 46, no. 4, pp. 467-475, 2003.

[23] H. B. Song, "Researches on dynamic evolution of gas hydrate system (II): submarine slides," Progress in Geophysics, vol. 18, no. 3, pp. 374-383, 2003.

[24] G. X. Zhang, H. N. Xu, and X. W. Liu, "The acoustic velocity characteristics of sediment with gas hydrate revealed by integrated exploration of 3D seismic and OBS data in Shen area," Chinese Journal of Geophysics, vol. 57, no. 4, pp. 1169-1176, 2014.

[25] X. J. Wang, J. P. Jin, Y. Q. Guo et al., "Enrichment characteristics and quantitative evaluation of natural gas hydrate in the northern South China Sea," vol. 46, no. 3, pp. 1038-1057, 2021.

[26] P. B. Su, J. Q. Liang, Z. J. Zhang, and Z. B. Sha, “Analysis of "bright spots" and "dark spots" on the seismic reflection profile of diffusion hydrates in the Shenhu sea area," Earth Science Frontiers, vol. 24, no. 4, pp. 51-56, 2017.

[27] S. S. Shi, X. Z. Chen, and J. Ma, "Natural gas hydrate reservoir classification and characterization in the well W19 of Shenhu area, northern South China Sea," Special Oil \& Gas Reservoirs, vol. 26, no. 3, pp. 24-29, 2019.

[28] J. P. Jin, "Geological controls on the occurrence of recently formed highly concentrated gas hydrate accumulations in the Shenhu area, South China Sea," Marine and Petroleum Geology, vol. 116, 2020.

[29] C. F. Li, G. W. Hu, W. Zhang, and Y. G. Ye, "The influence of foraminifera on the formation and occurrence of natural gas hydrate in fine-grained sediments in the Shenhu area of the South China Sea," Scientia Sinica Terrae, vol. 9, pp. 12231230, 2016.

[30] J. T. Ahrens, "Rock physics \& phase relations: a handbook of physical constants volume 3 thermal conductivity of rocks and minerals," American Geophysical Union, pp. 105-126, 1995.

[31] R. Ciz and S. A. Shapiro, "Generalization of Gassmann equations for porous media saturated with a solid material," Geophysics, vol. 72, pp. 75-79, 2007.
[32] X. X. Liu, X. Y. Yin, and X. W. Luan, "Construction of petrophysical model of gas hydrate formation," Science China: Earth Science, vol. 48, no. 9, pp. 128-146, 2018.

[33] J. Q. Liang, G. G. Zhang, J. G. Lu, P. B. Su, Z. B. Sha, and Y. H. Gong, "Natural gas hydrate enrichment characteristics and genetic model on the northeastern slope of the South China Sea," Natural Gas Industry, vol. 36, no. 10, pp. 157-162, 2016. 